\title{
HMGB1 promotes ductular reaction and tumorigenesis in autophagy-deficient livers
}

\author{
Bilon Khambu, ${ }^{1}$ Nazmul Huda, ${ }^{1}$ Xiaoyun Chen, ${ }^{1}$ Yong Li, ${ }^{1}$ Guoli Dai, ${ }^{2}$ Ulrike A. Köhler, ${ }^{3}$ Wei-Xing Zong, ${ }^{4}$ \\ Satoshi Waguri, ${ }^{5}$ Sabine Werner, ${ }^{3}$ Tim D. Oury, ${ }^{6}$ Zheng Dong, ${ }^{7,8}$ and Xiao-Ming Yin ${ }^{1}$ \\ 'Department of Pathology and Laboratory Medicine, Indiana University School of Medicine, Indianapolis, Indiana, USA.'Department of Biology, Purdue University School of Science, Indianapolis, Indiana, \\ USA. ${ }^{3}$ Department of Biology, Institute of Molecular Health Sciences, Swiss Federal Institute of Technology (ETH) Zurich, Zurich, Switzerland. ${ }^{4}$ Department of Chemical Biology, Ernest Mario School of \\ Pharmacy, Rutgers University, New Brunswick, New Jersey, USA. ${ }^{5}$ Department of Anatomy and Histology, Fukushima Medical University, School of Medicine, Fukushima, Japan. ${ }^{6}$ Department of Pathology, \\ University of Pittsburgh School of Medicine, Pittsburgh, Pennsylvania, USA. 'Department of Nephrology, The Second Xiangya Hospital, Central South University, Changsha, Hunan, China. ${ }^{8}$ Department of \\ Cellular Biology and Anatomy, Medical College of Georgia at Augusta University and Charlie Norwood VA Medical Center, Augusta, Georgia, USA.
}

\begin{abstract}
Autophagy is important for liver homeostasis, and the deficiency leads to injury, inflammation, ductular reaction (DR), fibrosis, and tumorigenesis. It is not clear how these events are mechanistically linked to autophagy deficiency. Here, we reveal the role of high-mobility group box 1 (HMCB1) in two of these processes. First, HMGB1 was required for DR, which represents the expansion of hepatic progenitor cells (HPCs) implicated in liver repair and regeneration. DR caused by hepatotoxic diets (3,5-diethoxycarbonyl-1,4-dihydrocollidine [DDC] or choline-deficient, ethionine-supplemented [CDE]) also depended on HMCB1, indicating that HMCB1 may be generally required for DR in various injury scenarios. Second, HMCB1 promoted tumor progression in autophagy-deficient livers. Receptor for advanced glycation end product (RAGE), a receptor for HMCB1, was required in the same two processes and could mediate the proliferative effects of HMBC1 in isolated HPCs. HMGB1 was released from autophagy-deficient hepatocytes independently of cellular injury but depended on NRF2 and the inflammasome, which was activated by NRF2. Pharmacological or genetic activation of NRF2 alone, without disabling autophagy or causing injury, was sufficient to cause inflammasome-dependent HMCB1 release. In conclusion, HMCB1 release is a critical mechanism in hepatic pathogenesis under autophagy-deficient conditions and leads to HPC expansion as well as tumor progression.
\end{abstract}

\section{Introduction}

Macroautophagy, hereafter referred to as autophagy, is an essential biological process for degrading cellular materials via the lysosome (1). Disruption of autophagy in various organs results in pathological changes that in some cases can be ascribed to a failure to degrade harmful cellular constituents (2), but in other cases are not fully understood mechanistically. Autophagy deficiency in the liver leads to hepatomegaly, cellular injury, inflammation, fibrosis, expansion of hepatic progenitor cells (HPCs) or ductular cells (DCs), and tumor development, the underlying mechanisms of which are still unclear. These pathological changes mirror those observed in other liver diseases with different etiologies. Thus, while the insulting events are different, the subsequent pathological changes may share common pathways. A commonly held notion is that cell death can lead to inflammation, or vice versa, and that this vicious cycle in a chronic disease course results in significant tissue injury, which is accompanied by a certain degree of tissue repair and fibrosis. In the cycle of destruction and regeneration, genomic instability can be induced, which can ultimately lead to cancer development $(3,4)$. However, the molecular signal-

Conflict of interest: The authors have declared that no conflict of interest exists. Submitted: November 17, 2016; Accepted: March 13, 2018.

Reference information: / Clin Invest. 2018;128(6):2419-2435.

https://doi.org/10.1172/JCI91814. ing events that individually regulate and/or globally connect these processes are not fully understood.

High-mobility group box 1 (HMGB1) is a damage/dangerassociated molecular pattern (DAMP) molecule $(5,6)$. HMGB1 is expressed in virtually all nucleated eukaryotic cells. It is mainly located in the nucleus but can also be found in the cytosol and the extracellular space. In the nucleus, fully reduced HMGB1 binds to DNA and facilitates DNA-protein interaction, gene transcription, and DNA replication and repair. Its extracellular function as a DAMP molecule is best studied in association with sepsis, tissue injury, inflammation, and fibrosis (5-10). The release of HMGB1 can be passive, from dead cells, but can also be active, from activated macrophages (11-13). Extracellular HMGB1 can recruit immune cells, induce cytokines and chemokines, and promote inflammation, angiogenesis, and tissue repair $(5,6,14)$.

Hepatic HMGB1 can participate in inflammation, fibrosis, and injury caused by hepatitis C virus (HCV) or $\mathrm{CCl}_{4}$ (7), ischemia/ reperfusion (15), acetaminophen (10), or ethanol (16). It was therefore reasonable to hypothesize that HMGB1 could participate in the liver pathology induced by autophagy deficiency. Indeed, in this study, we found that HMGB1 in hepatocytes translocated from the nucleus to the extracellular space in response to autophagy deficiency in the liver. We show that the molecular pathway that connects autophagy deficiency to the active release of HMGB1 is mediated via NRF2 and inflammasomes. Surprisingly, hepato- 
A
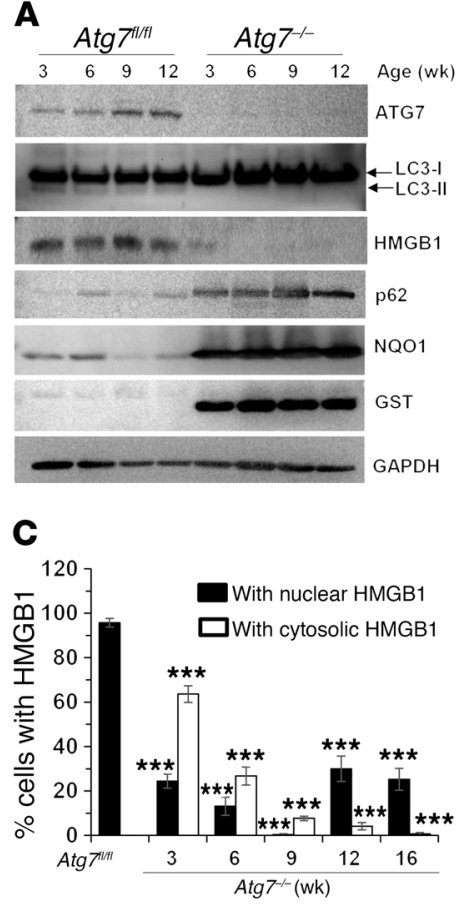

B

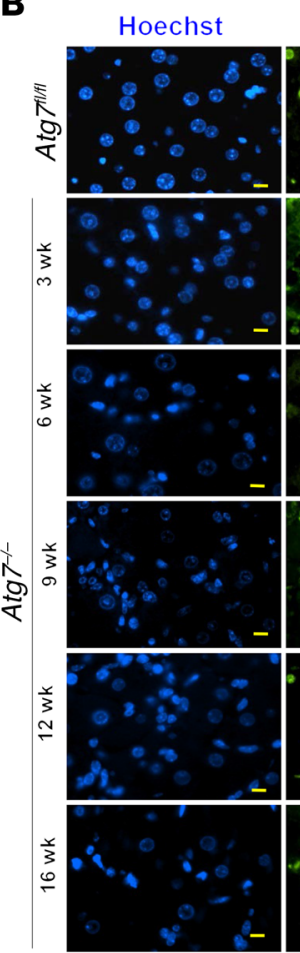

HMGB1
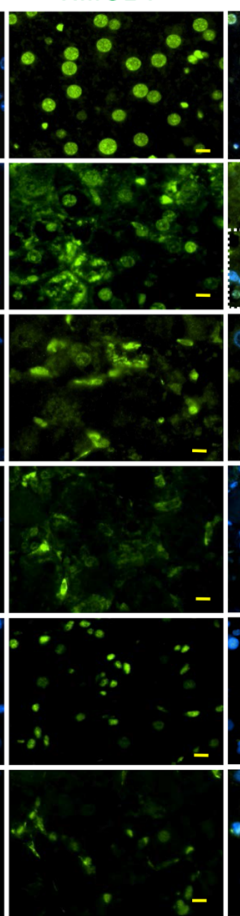

Merge
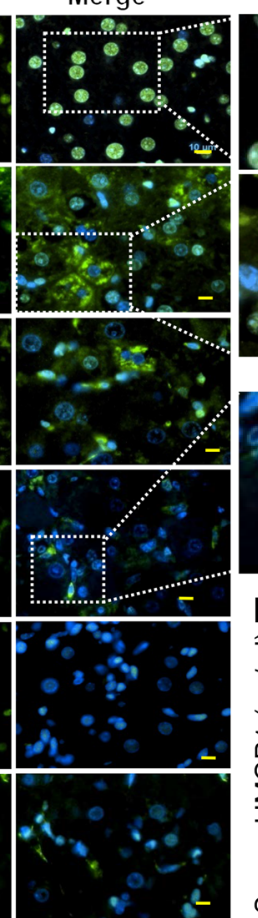
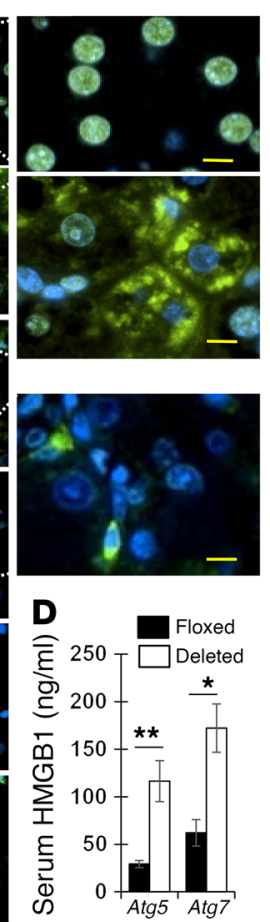

E

Hoechst-HMGB1

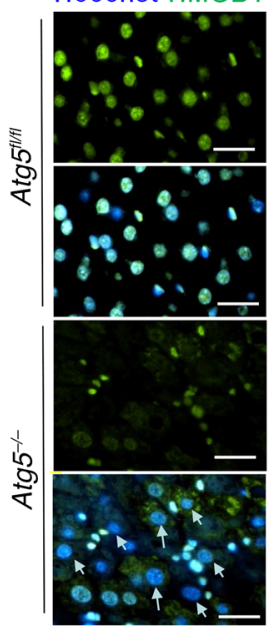

$\mathbf{F}$

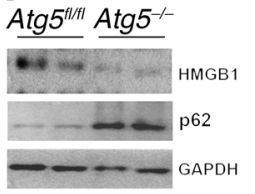

Figure 1. Hepatic autophagy deficiency causes HMGB1 release from hepatocytes. (A) Immunoblot analysis of hepatic lysates from Atg $7^{f / f l}$ and $A t g 7^{1 H e p}$ $\left(A t g 7^{-/}\right)$mice at the indicated ages. (B) Liver sections from 9-week-old Atg $7^{f l / f l}$ mice and Atg $7^{\Delta H e p}$ mice at different ages were immunostained for HMGB1. (C) Percentage of hepatocytes with nuclear or cytosolic HMGB1 ( $n=3$ mice/group). (D) Serum HMCB1 levels in 6-week-old mice ( $n=4-7$ mice/group). (E and F) Liver sections or lysates from 9-week-old $A t g 5^{f l f l}$ and $A t g 5^{1 H e p}\left(A t g 5^{-/-}\right)$mice were subjected to immunostaining (E) or immunoblot assay (F) for HMCB1. Arrows in E indicate hepatocytes without nuclear HMCB1. Scale bars: $10 \mu \mathrm{m}$. Data represent the mean \pm SEM. ${ }^{*} P<0.05$, ${ }^{* *} P<0.01$, and ${ }^{* * *} P<0.001$, by 1 -way ANOVA with Duncan's post hoc analysis (C) and 2-sided Student's $t$ test (D).

cyte-derived HMGB1 did not critically affect sterile inflammation or fibrosis, but promoted repair-associated DC/HPC expansion and tumor development in autophagy-deficient livers. These findings indicate that hepatocyte-derived HMGB1 can play important roles in liver pathology under autophagy-deficient conditions.

\section{Results}

Autophagy-deficient hepatocytes release HMGB1. In our investigation of the mechanism of liver injury under autophagy deficiency, we examined HMGB1 levels in the liver. Deletion of a key autophagy gene, $A t g 7$, in the liver $\left(A \operatorname{tg} 7^{\Delta \mathrm{Hep}}\right)$ caused loss of the lipidated form of LC3 (LC3-II), the accumulation of p62 (also known as SQSTM1), an adaptor molecule subjected to autophagy degradation, and the activation of NRF2, leading to an increased expression of the NRF2 target genes Nqo1 and Gst in a time-dependent manner (Figure 1A). We found that the kinetics of HMGB1 protein level decline were similar (Figure $1 \mathrm{~A}$ ), with no changes in mRNA levels (Supplemental Figure 1A; supplemental material available online with this article; https:// doi.org/10.1172/JCI91814DS1). Other organs in Atg $7^{\text {Анер }}$ mice did not show HMGB1 reduction or translocation (Supplemental Figure 1, B and C). The loss or translocation of HMGB1 occurred in hepatocytes, but not in the nonparenchymal cells in the liver (Figure 1B), which was confirmed using isolated hepatocytes and nonhepatocytes (Supplemental Figure 1D).
HMGB1 signal was present in the hepatocyte nucleus of control Atg7-floxed (Atg $\left.7^{f l / f l}\right)$ livers. In 3-week-old Atg $7^{4 \text { Hep }}$ mice, HMGB1 was mainly found in the cytosol of the hepatocyte (Figure 1, B and C), indicating an enhanced nucleus-to-cytosol translocation. Over time, both nuclear and cytosolic HMGB1 were decreased in Atg $7^{-1-}$ hepatocytes, with the nadir occurring at 9 weeks of age. Concomitantly, we detected significantly elevated levels of HMGB1 in the $A t g 7^{\text {АHеp }}$ serum (Figure 1D), supporting the notion that HMGB1 loss in hepatocytes is due to its release. In older mice (12-16 weeks of age), approximately $20 \%$ of hepatocytes retained nuclear HMGB1 (Figure 1, B and C), perhaps reflecting the steady productionrelease dynamics of intracellular HMGB1.

We confirmed the loss of HMGB1 in hepatocyte nuclei in 2 other strains of hepatocyte-specific, autophagy-deficient mice: Atg5 $5^{\text {Анер }}$ (Figure 1, D-F) and Vps34 ${ }^{4 \text { Hер }}$ (Supplemental Figure 1, E and F). We also detected HMGB1 release from Atg7-deficient renal proximal tubules and a corresponding elevation of serum HMGB1 in this strain of mice (Supplemental Figure 1, G and H). These results suggest that an alteration of HMGB1 cellular location could be a common phenomenon in autophagy deficiency.

Hepatocyte-derived HMGB1 is important for DR, but not for inflammation or fibrosis. Autophagy-deficient livers manifest multiple pathological changes, including hepatocyte hypertrophy, inflammation, fibrosis, DR, and tumor development (17-20). To determine whether HMGB1 released from autophagy-deficient 
A

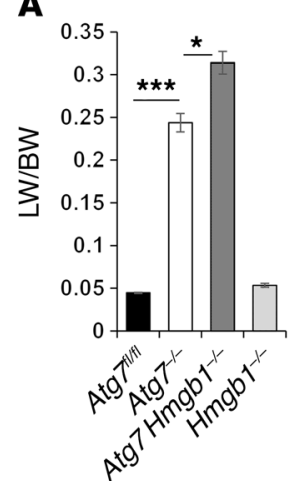

G
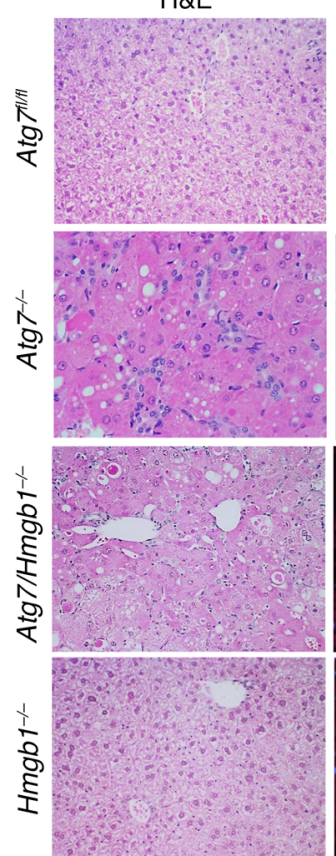

B

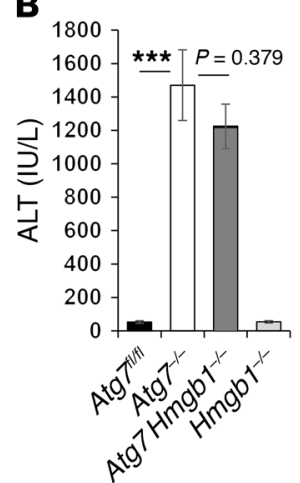

CK19-Hoechst
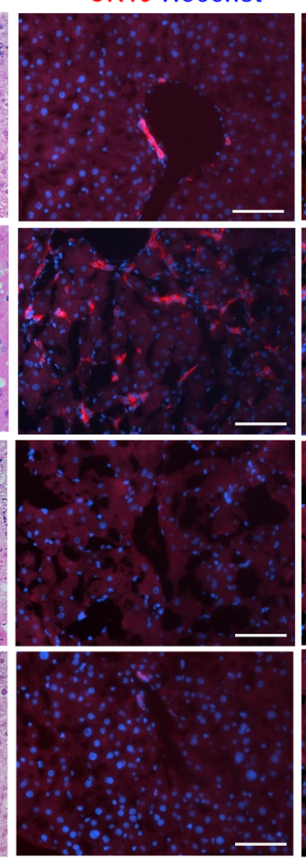

C

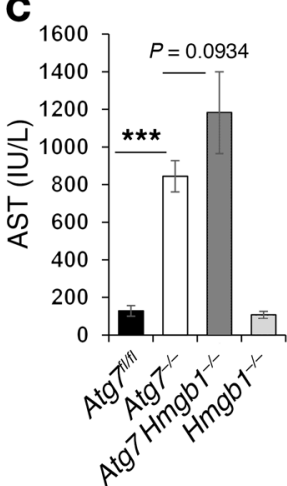

A6-Hoechst
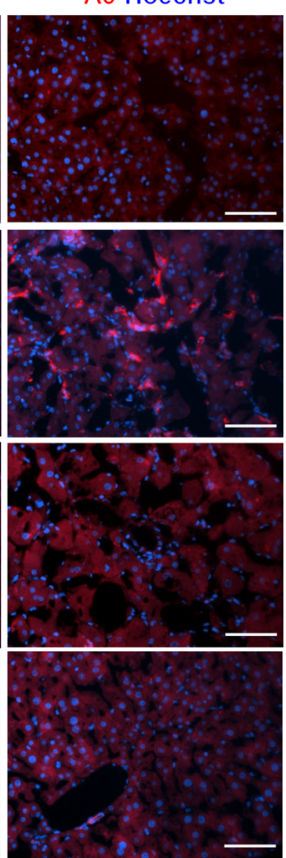

D

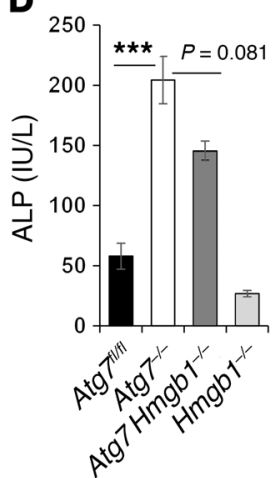

EpCAM-Hoechst

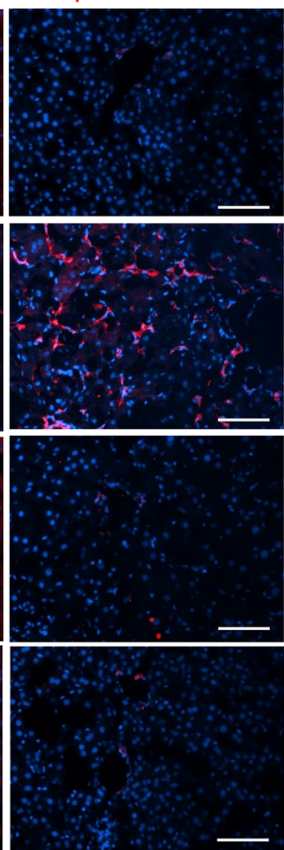

E

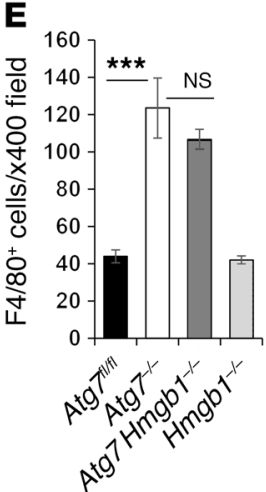

SOX9-Hoechst
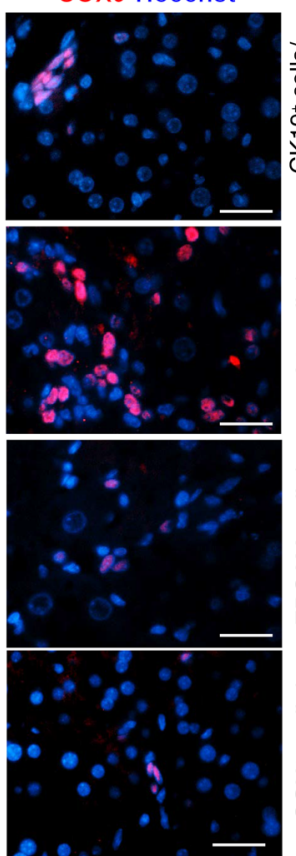

Figure 2. HMGB1 promotes DR in autophagy-deficient livers. (A-F) Mice were analyzed for liver weight (LW) versus body weight (BW) (A), for levels of serum ALT (B), AST (C), and ALP (D), the number of Kupffer cells (E), and the fibrotic area $(\mathbf{F})(n=4-7$ mice/group). (G and $\mathbf{H})$ Liver sections were subjected to H\&E staining (original magnification, $\times 200$ ) and immunostaining. DCs/ HPCs were quantified ( $n=3$ mice/group). Scale bars: $50 \mu \mathrm{m}$ (CK19, A6, and EPCAM) and $10 \mu \mathrm{m}$ (SOX9). Data represent the mean \pm SEM. ${ }^{*} P<0.05,{ }^{* *} P<0.01$, and ${ }^{* * *} P<0.001$, by 1 -way ANOVA with Duncan's post hoc analysis.

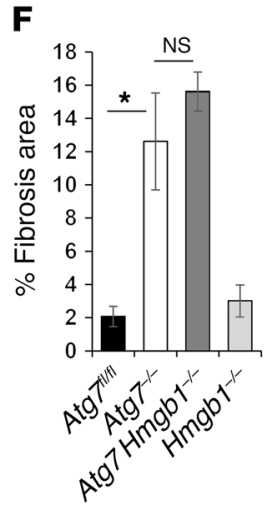

H
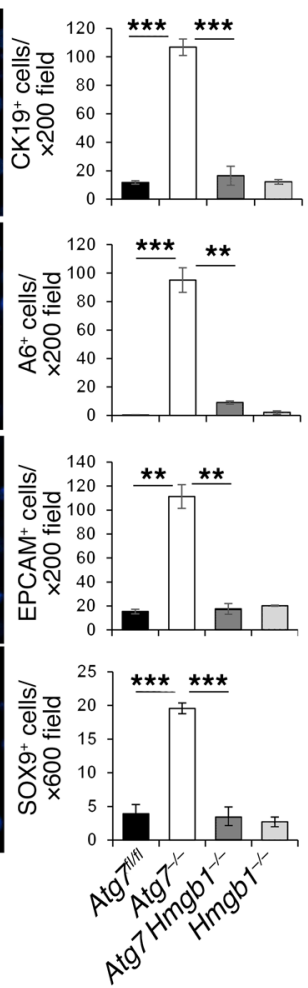

hepatocytes played a role in the phenotypic changes, we generated mice that were deficient in both Atg7 and Hmgb1 in hepatocytes

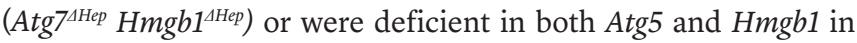
hepatocytes (Atg5 $5^{\text {AHep }} \mathrm{Hmgb1}^{\text {Hep }}$ ). Immunostaining for HMGB1 confirmed the absence of HMGB1 in hepatocytes, but not in nonhepatocytes in these mice (Supplemental Figure 2, A and B). We were consistently unable to detect HMGB1 in the sera of $A \operatorname{tg} 7^{\text {Aне }}$ $H m g b 1^{\text {HHep }}$ mice (Supplemental Figure 2C).

Codeletion of Hmgb1 in autophagy-deficient livers did not reduce NRF2 activation or p62 accumulation, but elevated hepatomegaly and hepatic injury (Figure 2, A-D, Supplemental Figure 3A, and Supplemental Figure 4, A and B). Inflammation in autophagy-deficient livers was evident by the expansion of $\mathrm{F} 4 / 8 \mathrm{O}^{+}$macrophages, $\mathrm{CD} 3^{+} \mathrm{T}$ cells, and $\mathrm{CD} 45 \mathrm{R}^{+} \mathrm{B}$ cells, but not $\mathrm{MPO}^{+}$neutrophils (Figure 2E, Supplemental Figure 3B, and Supplemental Figure 4C). We observed no obvious differences in the numbers of $\mathrm{F} 4 / 80^{+}$macrophages or other immune cells between $A t g 7^{1 \mathrm{Hep}}$ or Atg $5^{\text {AHep }}$ mice and their littermates with deletion of Hmgb1. Fibrosis was clearly detected in autophagy-deficient livers by trichrome or Picrosirius red staining (Supplemental Figure 3C and Supplemental Figure 4C). We also observed a notable increase in desmin ${ }^{+}$stellate cells, as well as increases in $\alpha-$ hydroxyproline, the expression of fibrogenic genes (Col1a1 and $C \operatorname{tg} f)$ and $\alpha$-smooth muscle actin ( $\alpha$-SMA) (Supplemental Figure $4, \mathrm{~A}$ and $\mathrm{C}-\mathrm{F}$ ), and increases in fibrotic area (Figure 2F). However, codeletion of $\mathrm{Hmgb1}$ in autophagy-deficient livers did not alter these changes. These results suggest that hepatic HMGB1 was dispensable for the development of inflammation and fibrosis in autophagy-deficient livers.

A prominent pathological change in the autophagy-deficient liver is the ductular reaction (DR), which, to our knowledge, has not been detailed in previous studies. We found that codeletion 
A

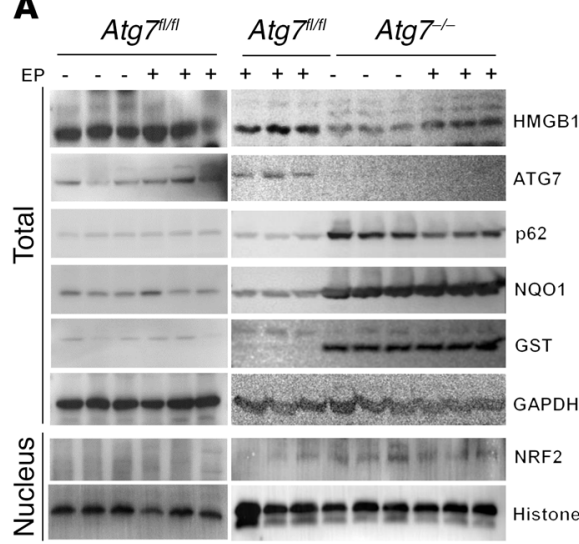

B

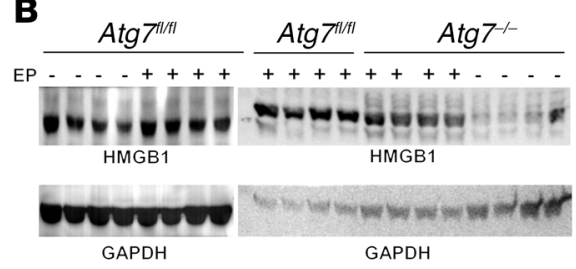

D

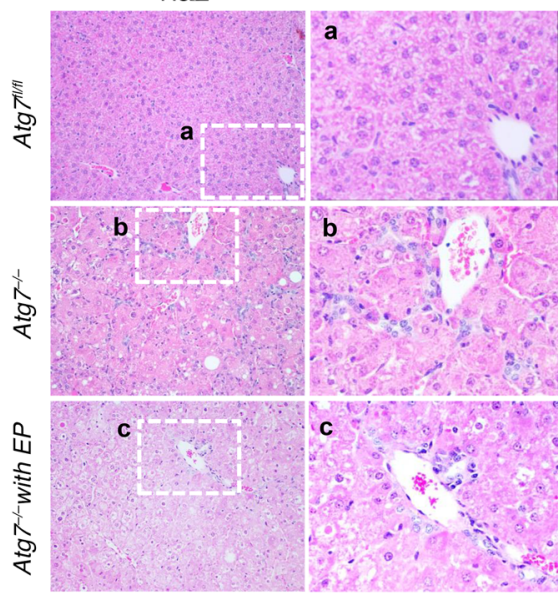

C
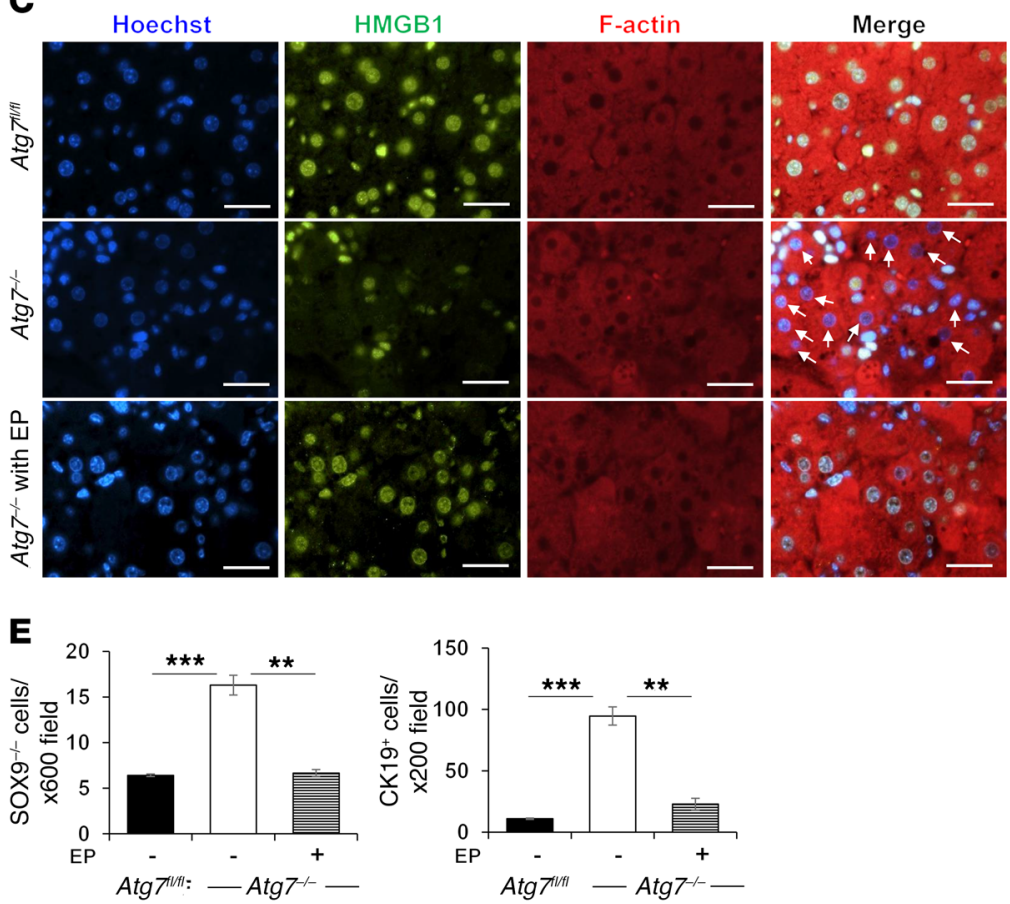

SOX9-Hoechst

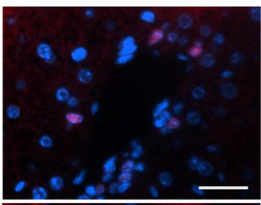

CK19-Hoechst

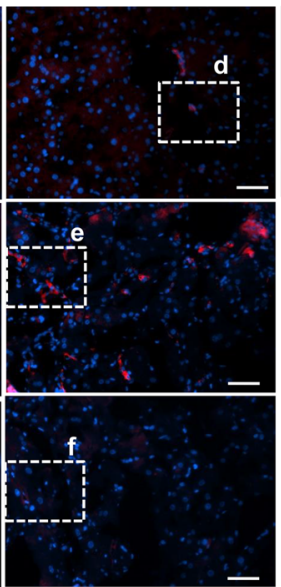

$\mathrm{F} 4 / 80$

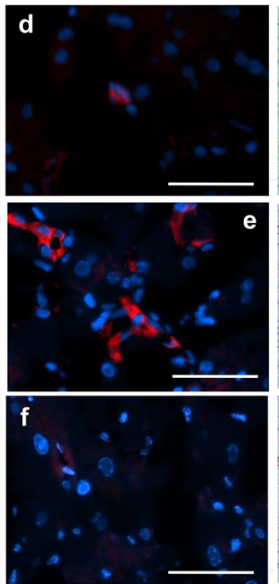

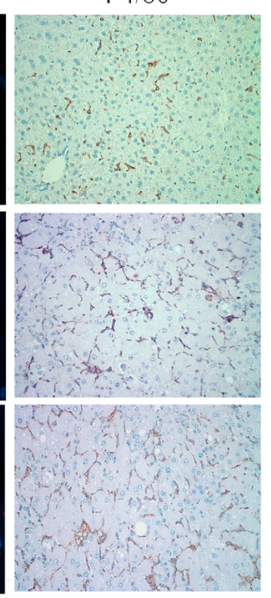

Figure 3. Pharmacological inhibition of HMGB1 release prevents DR. (A and B) Two-week-old mice were given EP i.p. (40 mg/kg) or the vehicle control twice a week for 2 (A) or 4 (B) weeks, and then analyzed 2 weeks later. Liver lysates and the nuclear fraction were examined by immunoblot assay. (C) Liver sections were stained as indicated. White arrows indicate nuclei that lost HMGB1 in Atg $7^{-/-}$without EP treatment. (D and E) Liver sections were H\&E stained (original magnification, $\times 200$ ) or immunostained as indicated (D). The framed areas are enlarged in separate panels. SOX9+ and CK19+ cells were quantified (E) ( $n=3$ mice/group). Scale bars: $10 \mu \mathrm{m}$ (C and SOX9 in D) and $50 \mu \mathrm{m}$ (CK19 in D). Original magnification, $\times 200$ (F4/80 in D). Data represent the mean \pm SEM. ${ }^{* *} P<0.01$ and ${ }^{* * *} P<0.001$, by 1-way ANOVA with Duncan's post hoc analysis.

of $H m g b 1$ in $A t g 7^{4 H e p}$ or Atg $5^{\text {AHep }}$ mice affected DR (Figure 2, G and $\mathrm{H}$, and Supplemental Figure 4D). DR is the expansion of DCs, also known as HPCs or oval cells (21-23). Under H\&E staining, these cells are small in size, with a high nucleus/cytoplasm ratio, and are distributed in a chain or clustered pattern (Figure $2 \mathrm{G}$ and Supplemental Figure 4D). Phenotypically, murine DCs/HPCs express mainly markers of biliary epithelial cells (21-23). Thus, we observed a significant increase in cells positive for CK19, A6, SOX9, or EPCAM in Atg $7^{4 H e p}$ and Atg $5^{\Delta H e p}$ livers (Figure 2, G and H, and Supplemental Figure 4D). DCs/HPCs were almost complete-

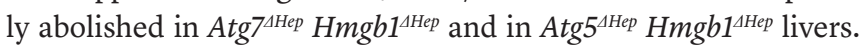

These results indicate that $\mathrm{HMGB} 1$ was required for the expansion of DCs/HPCs in autophagy-deficient livers. We found that DR

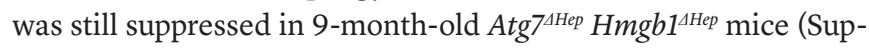
plemental Figure 5), indicating the long-term dependence of DCs/ HPCs on HMGB1 for expansion, which could not be compensated by other mechanisms.

We also assessed whether DR also depended on HMGB1 in liver injury caused by toxic diets. The 3,5-diethoxycarbonyl-1,4dihydrocollidine-supplemented (DDC-supplemented) diet (24) and the choline-deficient, ethionine-supplemented (CDE-supplemented) diet (25) can cause liver injury with significant DR. Indeed, 
A

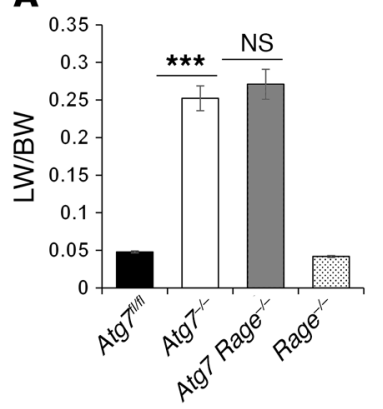

B

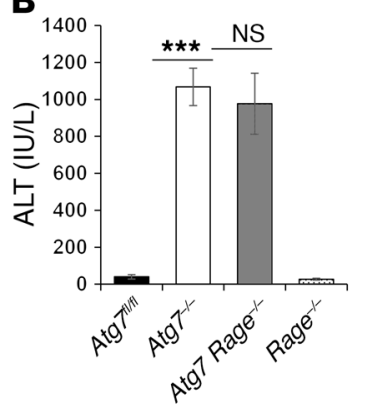

C

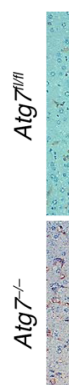

$\mathrm{F} 4 / 80$

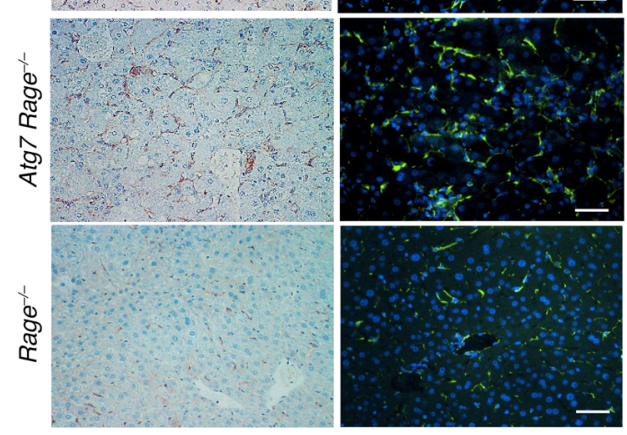

Desmin-Hoechst
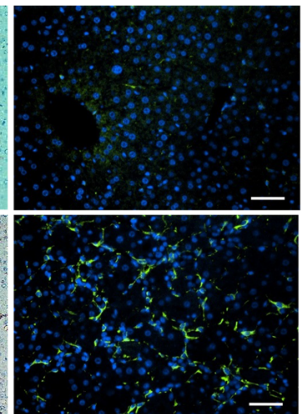

D

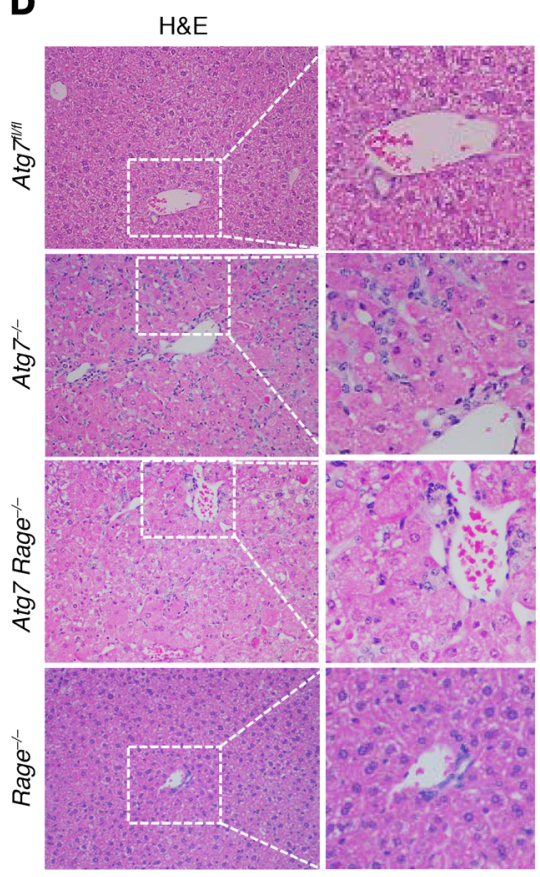

E
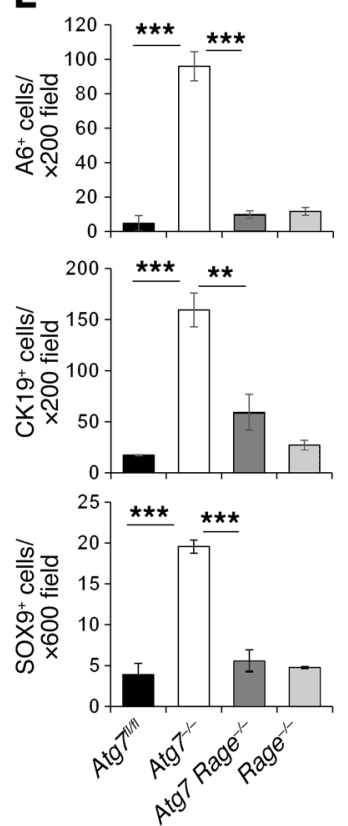

$\mathbf{F}$
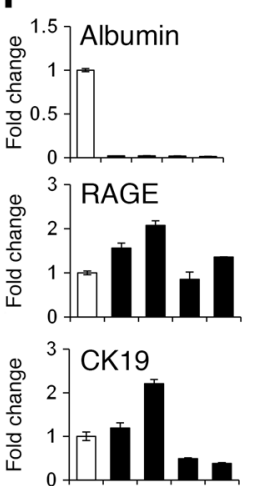

$\left.{ }^{1.5}\right] \alpha-S M A$

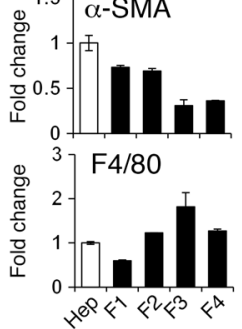

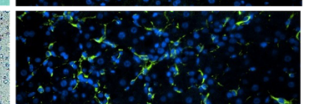

G
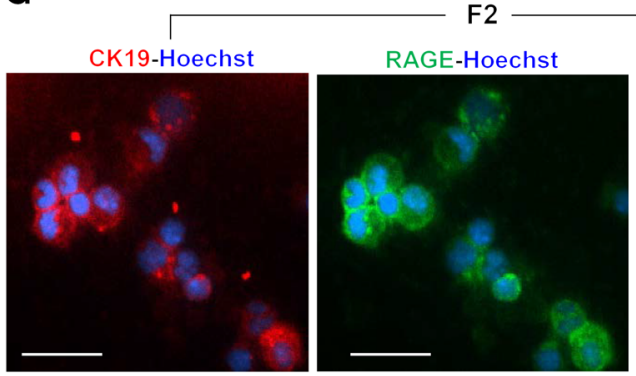

H

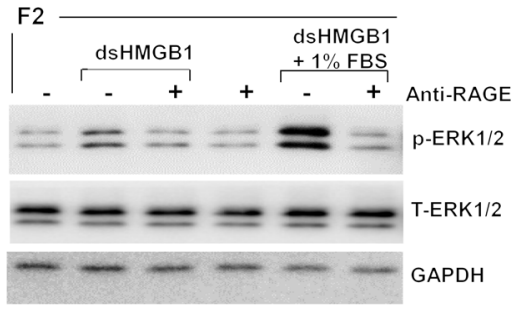

CK19-RAGE-Hoechst
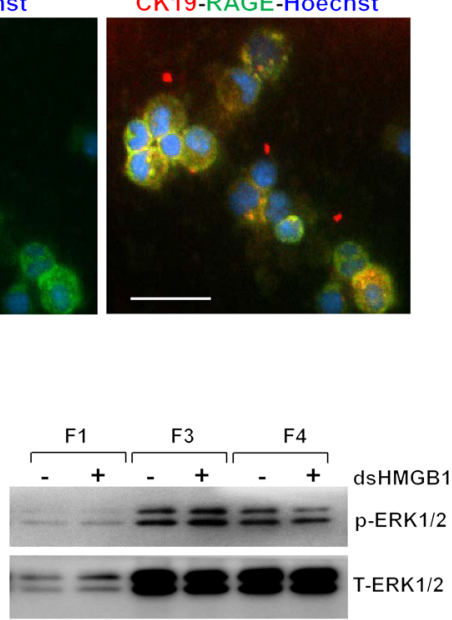

Figure 4. RAGE is required for DR in autophagy-deficient livers. (A and B) LW/BW and serum ALT levels were determined for $9-$ week-old mice ( $n=3-6$ mice/group). (C and D) Liver sections were stained for F4/80 or desmin (C, original magnification, $\times 200)$ or H\&E (D, original magnification, $\times 200)$. Framed areas in $\mathbf{D}$ were enlarged and are shown on the right. (E) Quantification of $\mathrm{CK}_{19^{+}}, \mathrm{A6}^{+}$, and SOX9+ cells ( $n=3$ mice/group). Images are shown in Supplemental Figure 8C. (F and G) Hepatic nonparenchymal cells were isolated from Atg7 ${ }^{\text {Hep-ERT2 }}$ mice $70-100$ days after tamoxifen treatment and were further fractioned on a Nycodenz density gradient. Quantitative real-time PCR (qRT-PCR) was performed on the 4 fractions (F1-F4) and hepatocytes (Hep). F2 cells were immunostained with anti-CK19 and anti-RACE. Data are representative of 2 experiments. (H) F2 (1.7 × $10^{5}$ cells/well) and F1 ( $2 \times 10^{5}$ cells/well) cells and F3 and F4 cells ( $5 \times 10^{5}$ cells/well) were cultured overnight and treated as indicated for 2 hours. Disulfide HMGB1: 60 ng/ml; anti-RAGE antibody: $2 \mu$ g/ml. T-ERK1/2, total ERK1/2; p-ERK1/2, phosphorylated ERK1/2. Scale bars: $50 \mu \mathrm{m}$ (C) and $10 \mu \mathrm{m}$ (G). Data represent the mean \pm SEM. ${ }^{*} P<0.01$ and ${ }^{*}{ }^{*} P<$ 0.001 , by 1-way ANOVA with Duncan's post hoc analysis.

DDC and CDE diets both induced HMGB1 release from hepatocytes (Supplemental Figure 6, A-D, and Supplemental Figures 7, A and B). Furthermore, DR, as measured by the level of CK19+ or $\mathrm{A}^{+}$cells or by $\mathrm{H} \& \mathrm{E}$ staining (Supplemental Figures 6, E-I, and
Supplemental Figure 7, C and D), was significantly suppressed in $H m g b 1^{\text {HHep }}$ livers, with no changes in liver injury (Supplemental Figures 6J). These results confirmed that HMGB1 was also required for the expansion of DCs/HPCs in response to a DDC or CDE diet. 
A

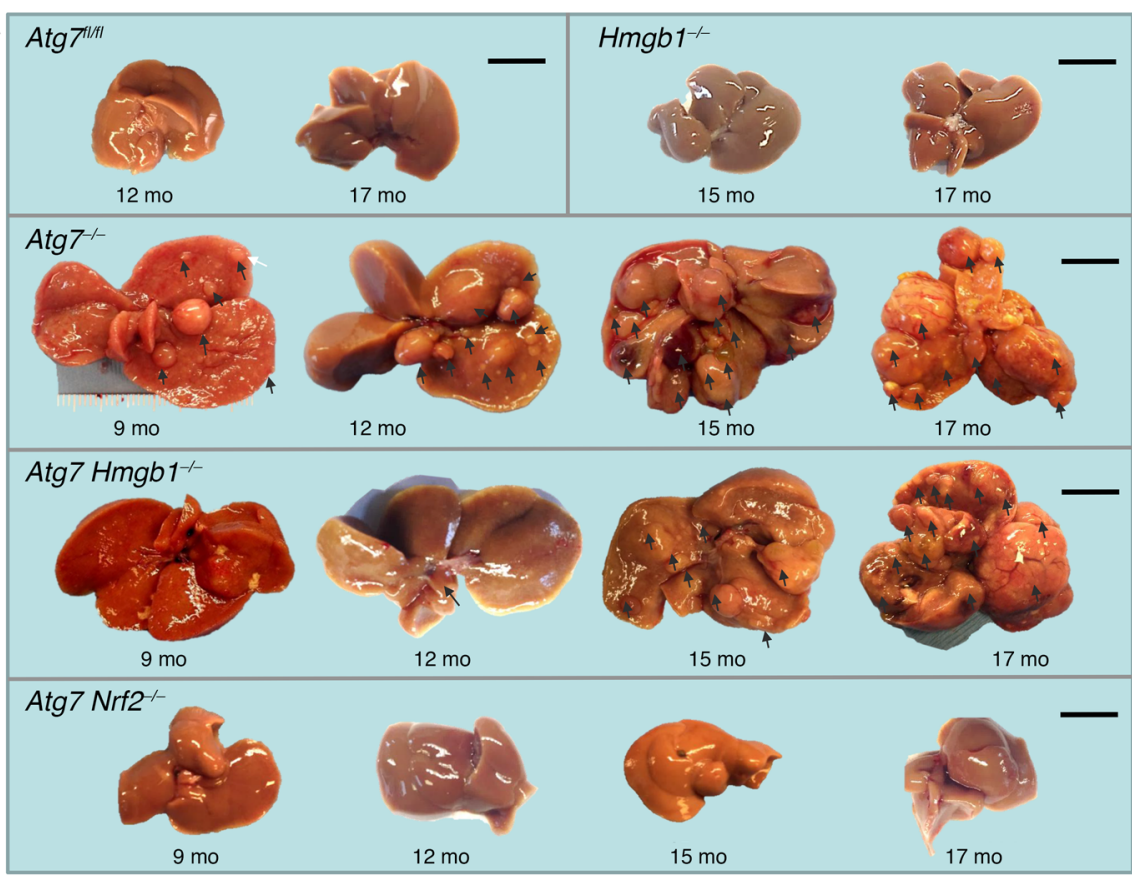

B

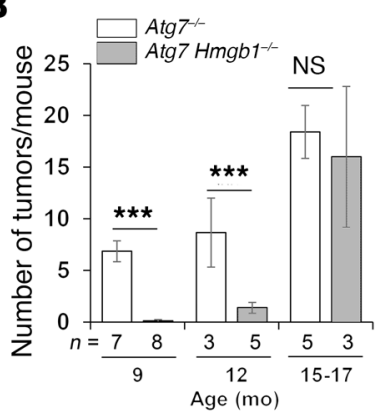

C.

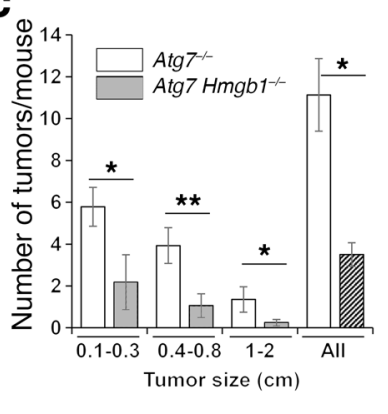

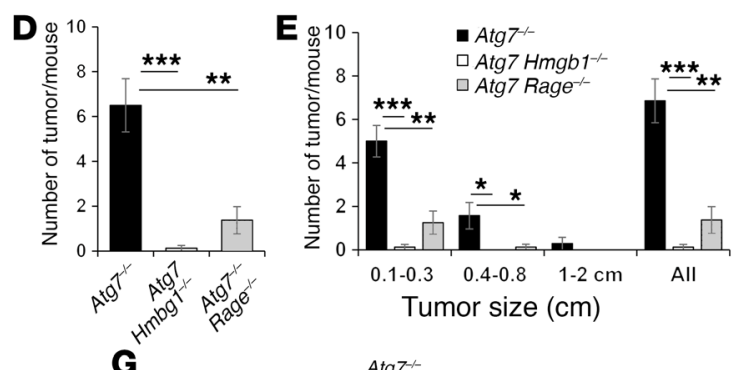

G
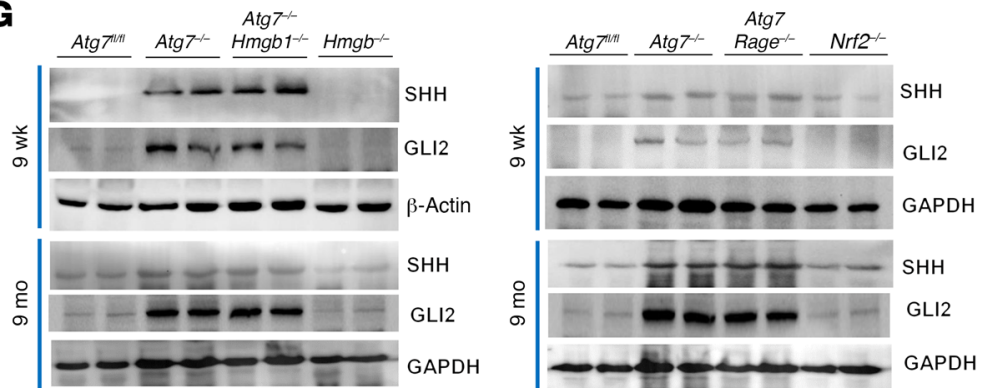

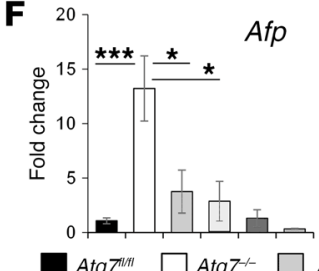

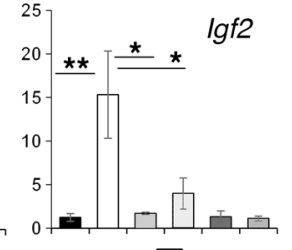

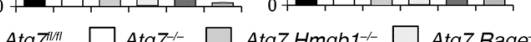

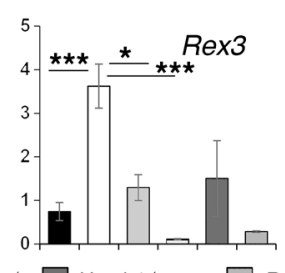

$\square \mathrm{Hmgb1}^{-1-}$

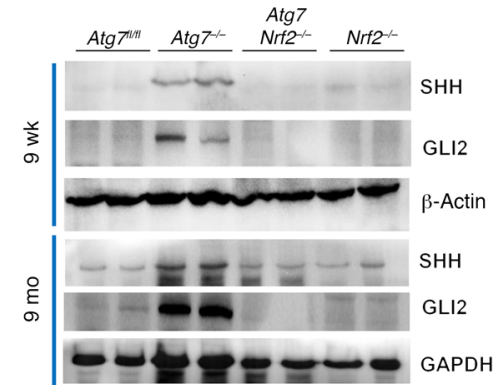

I GLI2-Hoechst GLI2

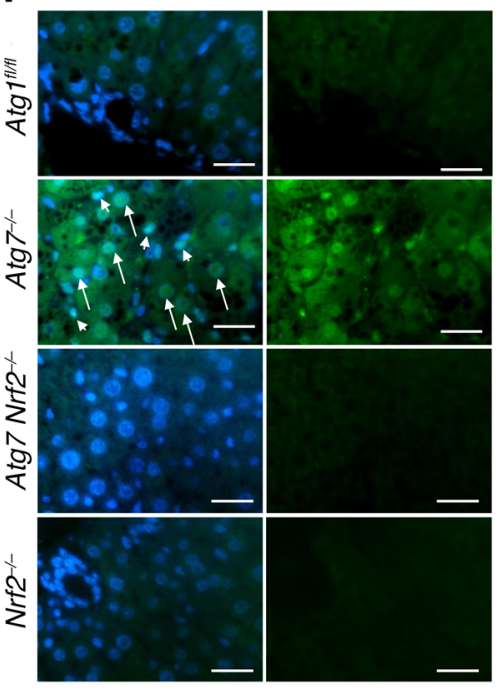


Figure 5. HMCB1 and RAGE promote tumorigenesis in autophagydeficient livers. (A) Gross observation of tumors (arrows) from 9- to 17-month-old mice. (B) Average number of tumors (by gross observation) in the livers of $A t g 7^{-/-}$and $A t g 7 ~ H m g b 1^{-/-}$mice. (C) Average size distribution of the tumors observed in $\operatorname{Atg}^{-{ }^{--}}(n=12)$ and Atg7 Hmgb7 $1^{-/-}(n=16)$ livers, with all age groups combined. ( $\mathbf{D}$ and $\mathbf{E}$ ) Average number and size distribution of tumors (by gross observation) in livers from 9-month-old mice $(n=$ 7-8 mice/group). (F) qRT-PCR analysis of hepatic expression of $A f p$, Igf2, and Rex3 in 9-month-old mice ( $n=3-6$ mice/group). (C) Immunoblot analysis of hepatic lysates from mice of the indicated ages. (H) Hepatic mRNA levels of Hedgehog signaling components in mice ( $n=3$ mice/group). (I) GLI2 staining of hepatic sections from 9-week-old mice. Arrows indicate GLI2 staining in hepatocytes, and arrowheads indicate GLI2 staining in nonparenchymal cells. Scale bars: $1 \mathrm{~cm}(\mathbf{A})$ and $10 \mu \mathrm{m}$ (I).Data represent the mean \pm SEM. ${ }^{*} P<0.05$, ${ }^{* *} P<0.01$, and ${ }^{* *} P<0.001$, by 1 -way ANOVA with Duncan's post hoc analysis ( $\mathbf{D}-\mathbf{F}$ and $\mathbf{H})$ and by 2-sided Student's $t$ test (B and $\mathbf{C})$.

HMGB1 acts extrinsically via its receptor for RAGE to promote $D R$. To determine whether HMGB1 promotes DR as an extrinsic mechanism, we treated 2-week-old Atg $7^{4 \mathrm{Hep}}$ mice with ethyl pyruvate (EP) twice a week for 2 or 4 weeks (Figure 3, A and B). EP is a potent lipophilic aliphatic ester of pyruvic acid, and it has been shown to inhibit HMGB1 release in sepsis $(26,27)$. EP was well tolerated and did not change autophagy deficiency, as p62 accumulation, nuclear translocation of NRF2, and NRF2 activation were retained (Figure 3A). Hepatic HMGB1 release in $A t g 7^{4 H e p}$ mice was inhibited in mice that received EP for 2 or 4 weeks (Figure 3, A-C). Consistently, EP treatment significantly inhibited DR in $A t g 7^{4 H e p}$ livers and the expansion of DCs/HPCs (Figure 3, D and E). In contrast, F4/80 cell infiltration was not affected (Figure 3D). These results support the notion that hepatocyte-released HMGB1 could act extrinsically to promote the expansion of DCs, which was inhibited by EP via the suppression of HMGB1 release.

HMGB1 acts through agonist receptors including the receptor for advanced glycation end products (RAGE) and several TLRs (6). We detected a small increase in the expression of Rage mRNA in autophagy-deficient livers (Supplemental Figure 8A). Codeletion of Rage did not protect the autophagy-deficient mice from liver injury or hepatomegaly (Figure 4, A and B), nor did it seem to protect their livers against inflammation or stellate cell expansion (Figure 4C). In addition, Rage deletion alone or Rage codeletion in $A t g 7^{4 H e p}$ mice did not affect HMGB1 release from hepatocytes (Supplemental Figure 8B). However, codeletion of Rage resulted in inhibition of DR (Figure 4D) and a suppression of the expansion of $\mathrm{A6}^{+}, \mathrm{CK} 19^{+}$, or $\mathrm{SOX}^{+} \mathrm{DCs} / \mathrm{HPCs}$ (Figure 4E and Supplemental Figure 8C). Notably, Rage deletion also inhibited DDC diet-induced $\mathrm{CK}_{19}{ }^{+}$cell expansion (Supplemental Figure 6, E and F). These results thus indicated that hepatocyte-derived HMGB1 caused DC expansion extrinsically through RAGE-mediated signaling.

To further examine the role of RAGE in the process, we isolated the putative DCs/HPCs using Nycodenz density gradient centrifugation (24). The fraction 2 (F2) cells isolated using this protocol had been characterized as the DCs/HPCs that contribute to DR (24). We isolated the F2 cells from the livers of Atg $7^{\text {Hep-ERT2 }}$ mice 70-100 days after tamoxifen induction, when DR was well developed (see below). We found that these F2 cells were enriched for the expression of RAGE and CK19, but not for albumin, F4/80, or $\alpha$-SMA (Figure 4F). Immunostaining confirmed the strong presence of both RAGE and CK19 in these cells (Figure 4G). When treated with recombinant disulfide HMGB1 proteins, F2 cells, but not other fractions of cells (F1, F3, F4), showed enhanced phosphorylation of ERK1/2, which was blocked by anti-RAGE antibody (Figure 4H), suggesting that these F2 cells had a RAGE-dependent proliferative response to HMGB1. In addition, we examined the well-defined DC/HPC cell line BMOL (28), which expressed A6, CK19, and RAGE (Supplemental Figure 9A) and responded to recombinant disulfide HMGB1 with enhanced ERK1/2 phosphorylation in a dose- and time-dependent manner, which was blocked by the anti-RAGE antibody (Supplemental Figure 9, B-D). The enhanced effect of HMGB1 in the presence of serum was also suppressed by anti-RAGE in both F2 and BMOL cells (Figure $4 \mathrm{H}$ and Supplemental Figure 9D). Taken together, these data support the role of $\mathrm{HMGB} 1$ in promoting the proliferation of $\mathrm{RAGE}^{+} \mathrm{DCs} /$ HPCs in a RAGE-dependent fashion.

HMGB1 and RAGE promote tumorigenesis in autophagydeficient livers. The functional role of DR in liver disease is not very well understood. It has been postulated that DCs/HPCs may serve as a regenerating pool for hepatocytes and cholangiocytes during chronic liver injury (21-23). Beyond liver repair, DCs are also implicated in the cellular compartment susceptible to malignant transformation $(29,30)$, although direct evidence has not been found (31). HMGB1 has also been implicated in tumor development in the liver and other tissues $(32,33)$.

We therefore sought to determine whether there were any differences in tumor development between $\operatorname{Atg} 7^{\Delta \mathrm{Hep}}$ and $\mathrm{Atg} 7^{\Delta \mathrm{Hep}}$ $H m g b 1^{1 H e p}$ mice. Atg $7^{f / f l}$ or $H m g b 1^{1 H e p}$ mice did not develop tumors within the 17-month observation period, but we observed a notable number of hepatic tumors in $A t g 7^{4 \mathrm{Hep}}$ mice starting at the age of 9 months, a trend that increased steadily through 15 to 17 months (Figure 5, A-C). Gross examination revealed no tumors in the livers of $A t g 7^{\text {АНе }} \mathrm{Hmgb}^{\text {4Hep }}$ mice at 9 months of age (Figure 5, A and B). However, we observed tumors in these mice at 12 month of age, but in significantly reduced numbers. We combined the few mice that had survived to the ages of 15 and 17 months and found that the average number of tumors in $A \operatorname{tg} 7^{4 \text { Нер }} H m g b 1^{4 \text { Нер }}$ mice was only slightly lower than that in $A t g 7^{4 H e p}$ mice (Figure $5 \mathrm{~B})$. However, when the size of the tumors was controlled, there were fewer tumors in each of the 3 given size groups in $A \operatorname{tg} 7^{4 \mathrm{Hep}}$ $H m g b 1^{4 H e p}$ mice than there were in $A t g 7^{4 H e p}$ mice (Figure 5C). As in the case of DR, we found that deletion of Rage also inhibited tumor development in $\operatorname{Atg} 7^{4 H e p}$ livers. We observed a significant reduction in tumor numbers and size in $A \operatorname{tg} 7^{4 \mathrm{Hep}} \mathrm{Rage}^{-/-}$livers (Figure 5, $\mathrm{D}$ and $\mathrm{E})$. In addition, expression levels of the 3 oncofetal genes $A f p$, insulin-like growth factor 2 (Igf2), and reduced in expression 3 (Rex3) were significantly upregulated in $A t g 7^{4 \mathrm{Hep}}$ livers, but not

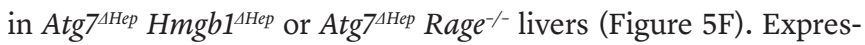
sion of these genes represented the development of dedifferentiated tumors (34). Finally, histological examination revealed welldemarcated $\mathrm{AFP}^{+}$hepatocellular adenomas in $A t g 7^{4 \mathrm{Hep}}$ livers (Supplemental Figure 10). There were significantly fewer tumor foci in $A t g 7^{4 \text { Hер }} \mathrm{Hmgb1}^{4 \mathrm{Hep}}$ livers, even at the age of 12 months. Expansion of tumor foci in $A t g 7^{\Delta H e p}$ livers was evident with reticulin staining. Interestingly, DCs and fibrotic responses were only present outside the tumor foci. Taken together, tumor development in 
A

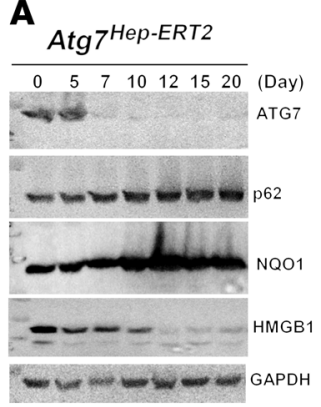

B

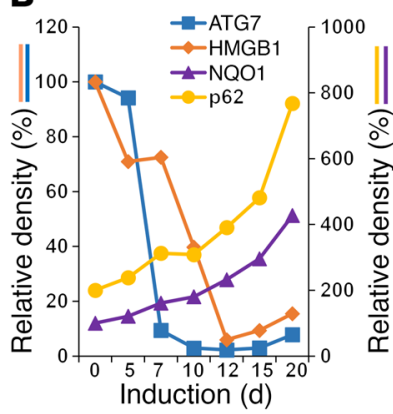

C

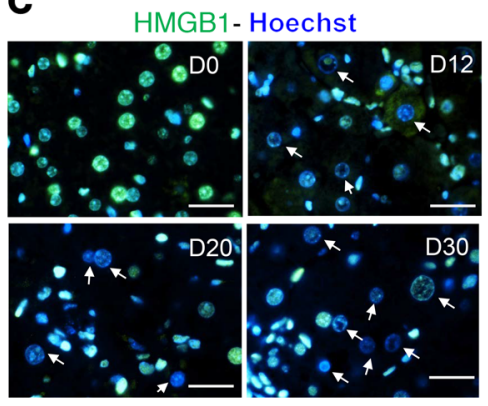

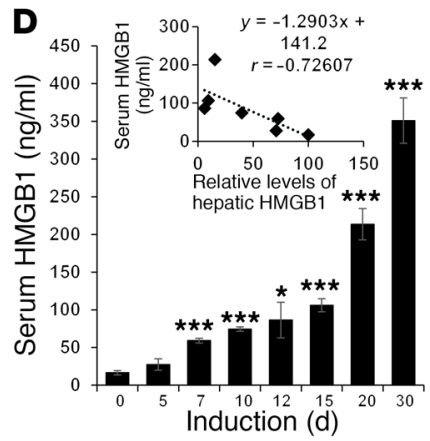

E

$\mathbf{F}$

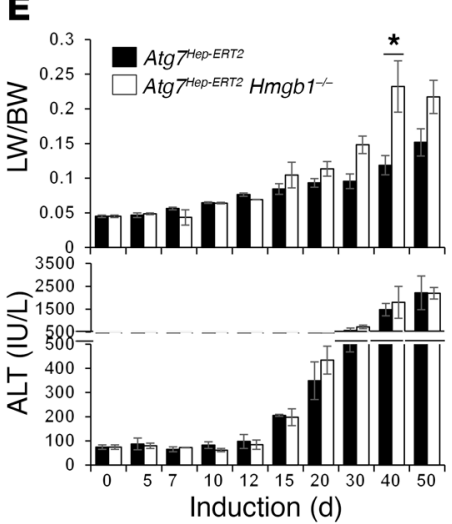

G

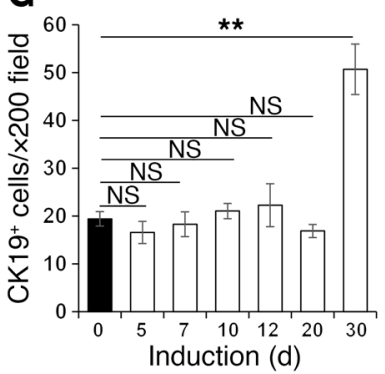

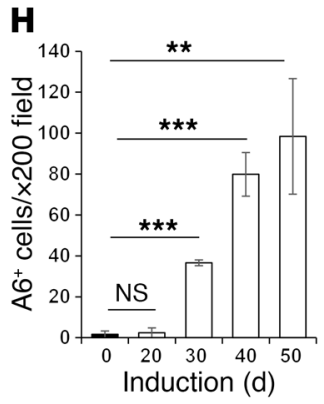
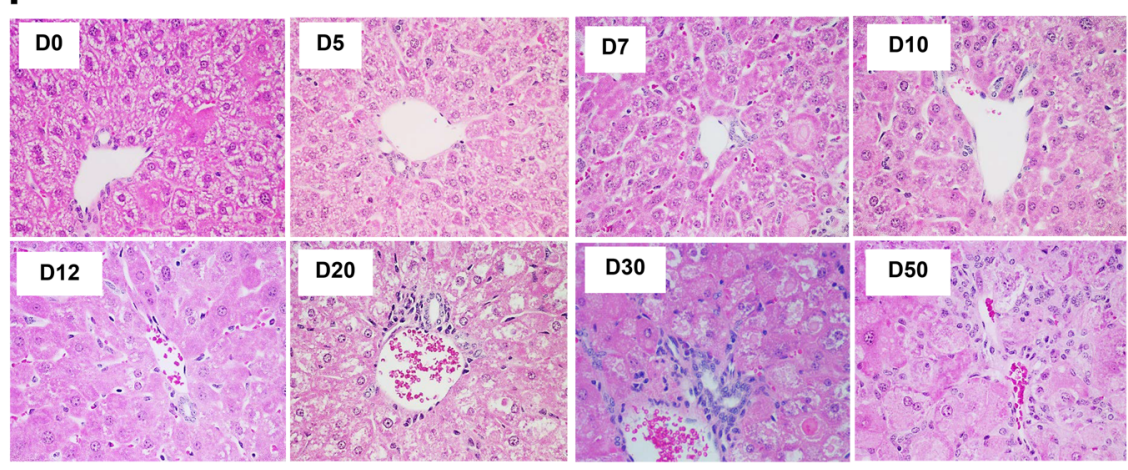
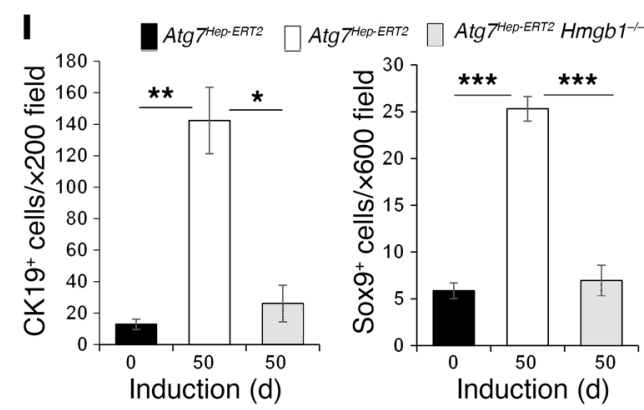

Figure 6. HMGB1 release is an early event following loss of autophagy function. (A) Atg $7^{\text {Hep-ERT2 }}$ mice were given tamoxifen on days 1 and 2. Livers were removed at different time points (days 5-20) and analyzed by immunoblot assay. Mice in the day 0 group received the vehicle control. (B) Densitometric

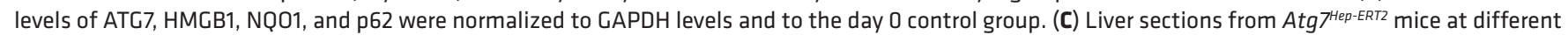
time points after induction were stained for HMGB1. Arrows indicate HMGB1-negative nuclei in hepatocytes. Scale bars: $10 \mu \mathrm{m}$. (D) Serum HMGB1 levels were determined in Atg7 $7^{\text {Hep-ERT2 }}$ mice from day 0 to day 30 after tamoxifen treatment. Significance was compared with day 0 ( $n=4$ mice/group). Insert shows the negative correlation of serum HMGB1 level with that of hepatic HMGB1 (from B). (E) Liver/body weight ratio (LW/BW) and serum ALT level were determined after tamoxifen treatment ( $n=3$ mice/group). Only statistically significantly different data are labelled with the asterisk (compared with day 0). (F) Liver sections from Atg ${ }^{\text {Hep-ERT2 }}$ mice at different time points after induction were H\&E stained. Original magnification, $\times 400$. (G and $\left.\mathbf{H}\right)$ The number of hepatic $\mathrm{CK}_{19}{ }^{+} \mathbf{( G )}$ and $\mathrm{A6}^{+}(\mathbf{H})$ cells was quantified in Atg $7^{\text {Hep-ERT2 }}$ mice at different time points after induction ( $n=3$ mice/group). See also the images in Supplemental Figure 12, E-F. (I) The number of hepatic CK19+ or SOX9+ cells was quantified in the indicated mice on days 0 and 50 after induction ( $n=3$ mice/group). See also the images in Supplemental Figure 12G. Data represent the mean \pm SEM. ${ }^{*} P<0.05$, ${ }^{* *} P<0.01$, and ${ }^{* * *} P<0.001$, by $1-$ way ANOVA with Duncan's post hoc analysis. DO, day 0; D5, day 5, etc.

autophagy-deficient livers was greatly affected by the events resulting from HMGB1-RAGE interaction.

While the data suggested that the HMGB1/RAGE axis might affect tumor progression, we also examined some of the molecular events that have been associated with the early tumorigenic process. Hedgehog signaling has been implicated in DR and cancer development. In $M d r 2$-deficient livers, which had developed strong DR and tumors (35), Hedgehog signaling was elevated, and its suppression inhibited DR and tumorigenesis (36). In Atg $7^{\text {AHep }}$ livers, we found that expression of $\mathrm{SHH}$, a ligand of the Hedgehog signaling pathway, was elevated (Figure 5G), while Hhip, coding for the inhibitory binding protein of $\mathrm{SHH}$, was downregulated (Figure 5H). HHIP downregulation is frequently seen in chronic liver injury, where Hedgehog signaling is elevated (37). mRNA expression levels of the $\mathrm{SHH}$ receptor patched 1 were moderately reduced, while mRNA levels of the activator Smo were moderately elevated (Figure $5 \mathrm{H}$ ). Since patched 1 works by inhibiting SMO, which is reversed by $\mathrm{SHH}$ binding, the detected changes in the expression of these genes favored the activation of SMO-mediated downstream events. Indeed, we found that 2 of the downstream 
A

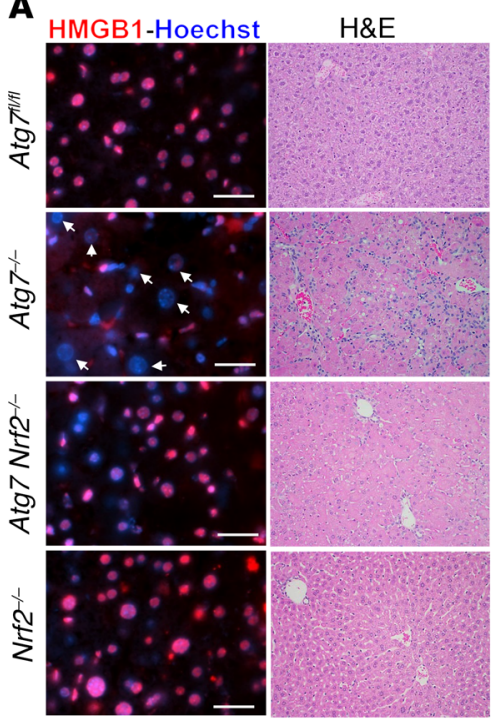

G

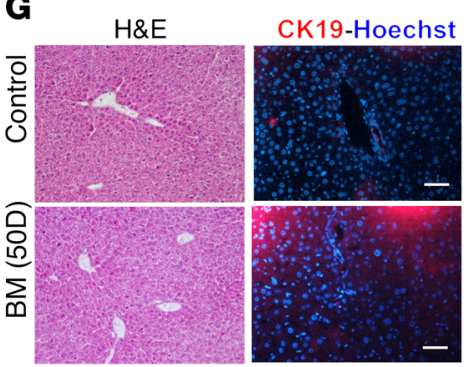

B

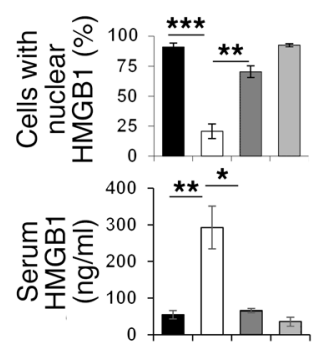

0
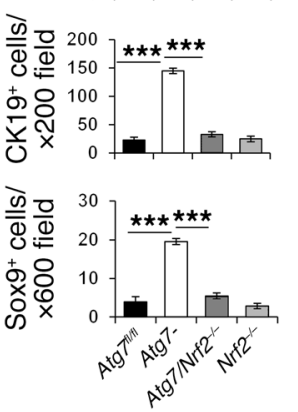

$\mathbf{H}$

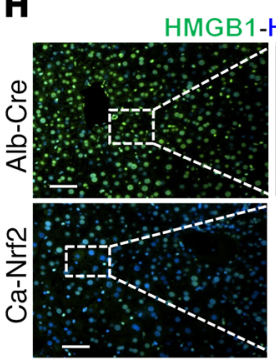

C
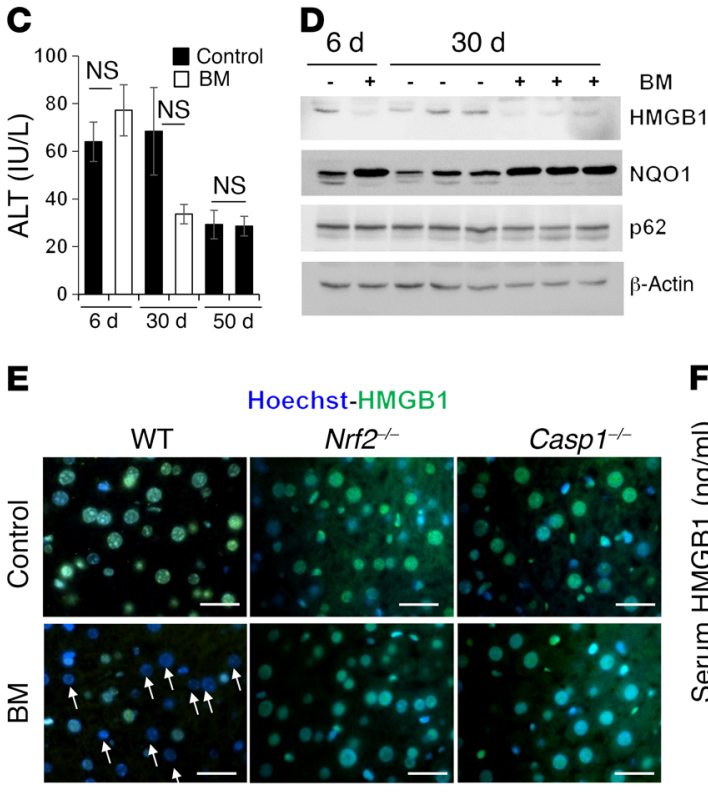

$\mathbf{F}$

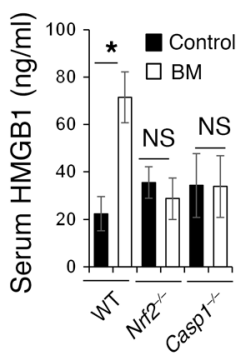

Figure 7. NRF2 is required for hepatic HMGB1 release and DR. (A) Liver sections of 9-week-old mice were H\&E stained (original magnification, $\times 200$ ) or stained for HMGB1. (B) Percentage of cells with nuclear HMGB1, serum levels of HMGB1, and number of CK19+ or SOX9+ cells ( $n=3-9$ mice/group). See Supplemental Figure 14D for images of CK19+ and SOX9+ staining. (C) WT mice were given the vehicle control or BM for different durations, and blood ALT levels were measured ( $n=3-6$ mice/group). Variations in ALT values for the control mice (50 days vs. 6 days and 30 days) were due to reagent lot changes. (D) WT mice were given the vehicle control or BM for different durations. Hepatic lysates were analyzed by immunoblot assay. (E) Liver sections of mice treated with BM or control for 6 days were stained as indicated. Arrows indicate hepatocytes without nucleus HMCB1. (F) Serum levels of HMGB1 ( $n=3-9$ mice/group). (G) Liver sections from mice receiving the 50 -day treatment regime were H\&E stained (original magnification, $\times 200$ ) or immunostained for CK19. (H and I) Liver sections from mice of the indicated genotypes were H\&E stained (original magnification, $\times 200$ ) or immunostained for HMCB1 or CK19 (H). Yellow arrows indicate hepatocytes without nuclear HMGB1; white arrows indicate hepatocytes with cytosolic HMGB1. (I) Cells with nuclear HMGB1 were quantified ( $n=3$ mice/group). Scale bars: $10 \mu \mathrm{m}(\mathbf{A}$ and $\mathbf{E})$ and $50 \mu \mathrm{m}$ (G and $\mathbf{H})$. Data represent the mean \pm SEM. ${ }^{*} P<0.05,{ }^{*} P<0.01$, and ${ }^{* * *} P<0.001$, by 1-way ANOVA (B) or Student's $t$ test (C, $\mathbf{F}$, and $\left.\mathbf{I}\right)$.

effectors, Gli1 at the transcriptional level (Figure $5 \mathrm{H}$ ) and GLI2 at the protein level (Figure 5, G and I), were elevated. We detected GLI2 at a molecular weight of $130 \mathrm{kDa}$, as previously reported in the liver (38). These changes were detected in mice at 9 weeks of age, before tumor development, and most of these changes remained detectable by 9 months of age, when tumors started to appear. These data suggest that the Hedgehog pathway was activated in autophagy-deficient livers at the early stage. However, codeletion of Hmgb1 or Rage did not significantly reverse these changes, suggesting that the HMGB1/RAGE axis might work in parallel with, but not contribute to, Hedgehog signaling events.

Autophagy deficiency-induced liver tumorigenesis was dependent on NRF2 signaling (Figure 5A) (39). In human HCVrelated hepatic cancers, NRF2-dependent tumorigenesis was found to be critically related to metabolic reprogramming in glutamate metabolism, the pentose phosphate pathway, purine nucleotide synthesis, and glucuronate metabolism (39). In Atg7deficient livers, we detected similar changes in the expression of key genes involved in these pathways (Supplemental Figure 11, A and B). These changes included an upregulation of Slc1a1 (a glutamate transporter) and Gclc (the catalytic subunit of glutamate-cysteine ligase), a key enzyme in glutathione synthesis, and a reduction of malic enzyme 1 (Me1), an enzyme important for the conversion of malate to pyruvate (Supplemental Figure 11C). In addition, we detected the upregulation of multiple genes in the pentose phosphate pathway as well as those for purine nucleotide synthesis and glucuronate metabolism (Supplemental Figure 11D). However, codeletion of Hmgb1 did not correct these changes, implying that HMGB1-mediated tumor progression did not involve the alterations of metabolic programming in this context.

HMGB1 is released soon after autophagy function declines. To understand how autophagy deficiency led to HMGB1 release, we 
A
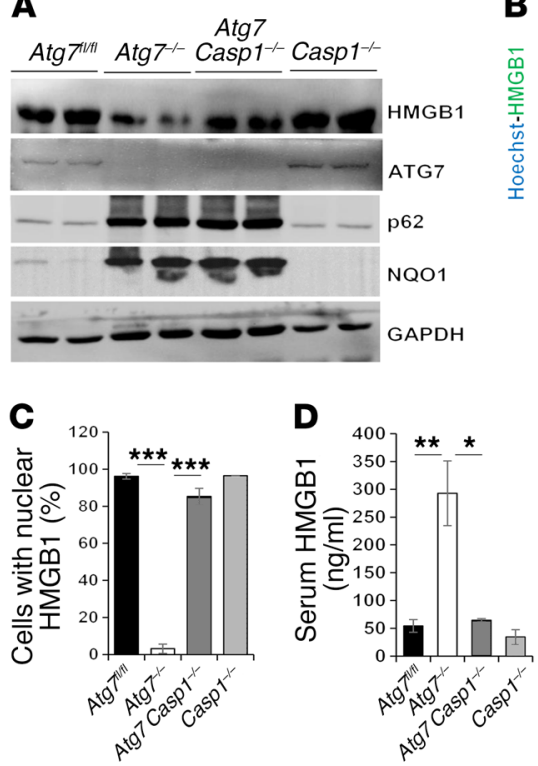

B
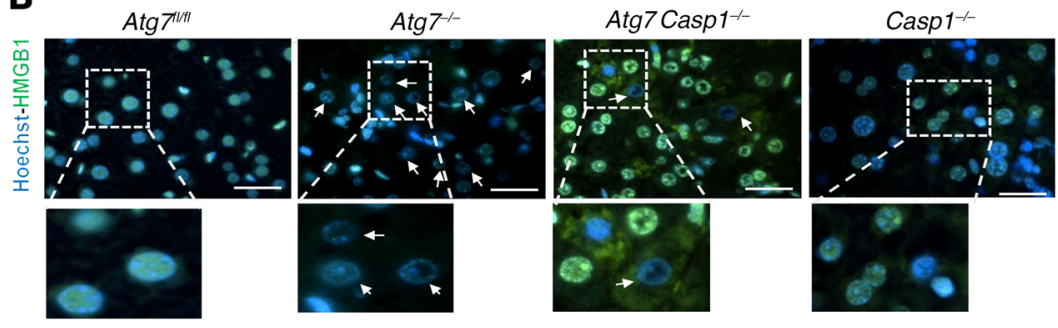

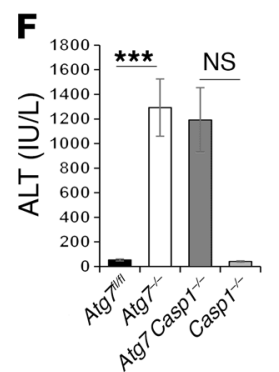

I

H

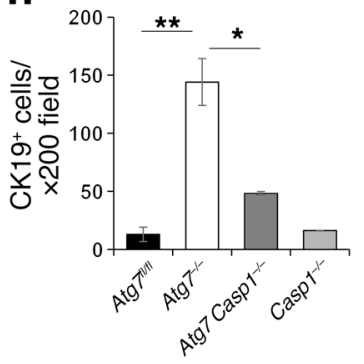

$\mathbf{K}$

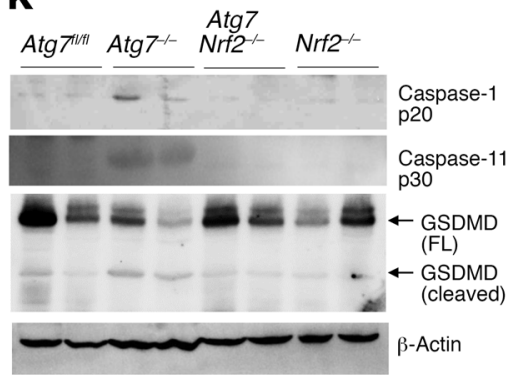

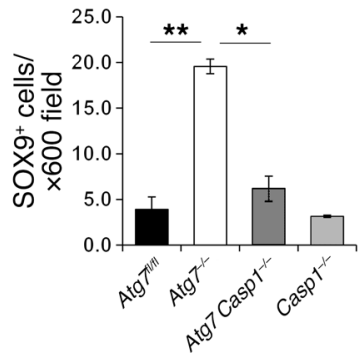

$\mathbf{L}$

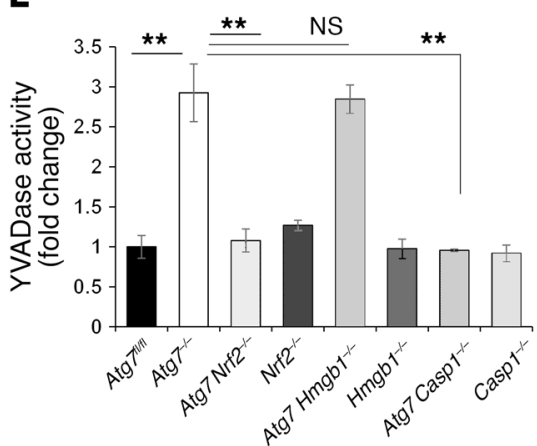

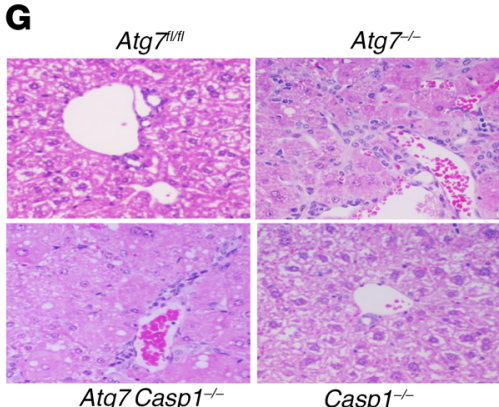

J

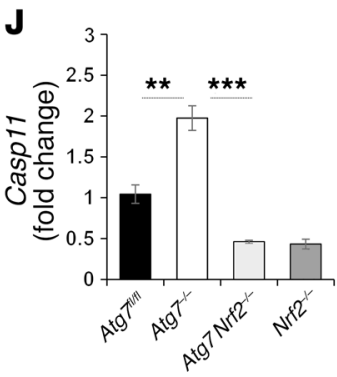

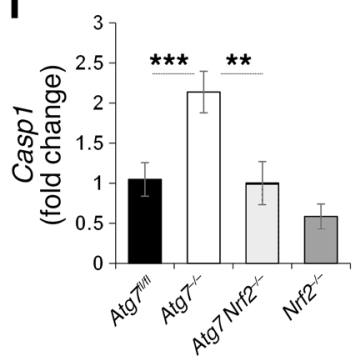

M

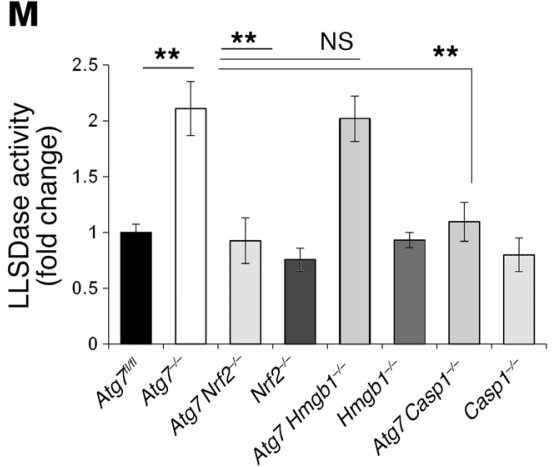
$\mathbf{N}$
Atg $7^{\wedge}$ or $\operatorname{Atg} 5^{-}$
(autophagy deficiency)
NRF2 activation

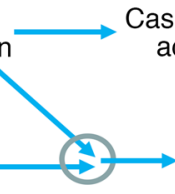

aspase-1/-11 activation

$-11$
$\longrightarrow \begin{gathered}\mathrm{HMGB} 1 \\ \text { release }\end{gathered}$

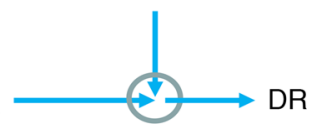

Both conditions are required 
Figure 8. The inflammasome is involved in HMGB1 release in autophagy-deficient liver. (A) Liver lysates from 9-week-old mice were analyzed by immunoblotting. (B) Liver sections were stained for HMCB1. Arrows indicate hepatocytes without nuclear HMGB1. Scale bars: $10 \mu \mathrm{m}$. (C) Cells with nuclear HMGB1 were quantified ( $n=3$ mice/group). (D) Serum levels of HMCB1 were measured. (E and $\mathbf{F}$ ) LW/BW ratios $(\mathbf{E})$ and serum ALT levels (F) were measured for 9-week-old mice. (C) Liver sections from 9 -week-old mice were H\&E stained. Original magnification, $\times 200$. (H) CK19+ and $\mathrm{SOX9}^{+}$cells were quantified. See also the images in Supplemental Figure 19A. (I and J) Hepatic mRNA levels of Casp1 (I) and Casp11 (J). Primer set 1 located in exon 6 was used for Casp11 amplification. (K-M) Liver lysates from 9-week-old mice were analyzed by immunoblotting or caspase activity assay ( $\mathbf{L}$ and $\mathbf{M})$. FL, full length. $(\mathbf{N})$ Model of the role of NRF2, inflammasomes, and HMGB1 in liver injury, DR, and tumor development. NRF2 activation is required and sufficient for caspase- 1 and caspase-11 activation and HMGB1 release in autophagy-deficient livers and is required, but may not be a sufficient condition, for autophagic liver injury or DR. Data represent the mean \pm SEM. $n=3-7$ mice/group. ${ }^{*} P<0.05,{ }^{* *} P<0.01$, and ${ }^{* * *} P<0.001$, by 1 -way ANOVA.

evaluated the temporal relationship of Atg7 deletion and HMGB1 release. We generated an inducible model of $A \operatorname{tg} 7$ deletion in the liver by crossing $A \operatorname{tg} 7^{f / / l}$ mice with $A l b-C r e-E R^{T 2}$ mice (40). The induction of Alb-Cre-ER ${ }^{\mathrm{T} 2}$ activity was carried out in adult mice, which resulted in loss of autophagy function in hepatocytes, but not in cholangiocytes.

The expression of ATG7 in the liver was largely absent 7 days after tamoxifen treatment of the inducible strain of mice (Figure $6 \mathrm{~A})$. We found that $\mathrm{p} 62$ and $\mathrm{NQO} 1$ levels were increased, indicating a loss of autophagy function and activation of NRF2. HMGB1 levels started to decline by day 5 , and by day 12 , immunoblot analysis revealed that HMGB1 was at approximately $6 \%$ of normal levels (Figure 6, A and B). We confirmed the loss of HMGB1 in hepatocytes by immunostaining (Figure 6C). Concomitantly, serum HMGB1 levels were progressively increased from day 5 (Figure 6D), which was in negative correlation with the hepatic levels of HMGB1. Early treatment of $A \operatorname{tg} 7^{\text {Hep-ERT2 }}$ mice with EP was able to inhibit HMGB1 release (Supplemental Figure 12, A and B). Notably, hepatomegaly and liver injury started on days 7 and 15, respectively, following Atg7 deletion in $A \operatorname{tg} 7^{\text {Hep-ERT2 }}$ mice (Figure $6 \mathrm{E})$. Thus, HMGB1 release was sensitive to the loss of autophagy function in hepatocytes (days 5-7) and was an early event in parallel with NRF2 activation (days 5-12), but earlier than the appearance of apparent liver injury.

DR was detected by day 30 and progressed subsequently (Figure $6 \mathrm{~F}$ ). The expansion of the CK- $19^{+}$and $\mathrm{A}^{+} \mathrm{DCs} / \mathrm{HPCs}$ was evident (Figure 6, G and H, and Supplemental Figure 12, C and D) and depended on HMGB1. Codeletion of Hmgb1 in Atg $7^{\text {Hep-ERT2 }}$ mice did not affect liver injury (Figure 6E), but did significantly reduce DR or the expansion of DCs/HPCs (Figure 6I and Supplemental Figure 12E). We observed hepatic tumors in $A \operatorname{tg} 7^{\text {Hep-ERT2 }}$ mice 12 months after tamoxifen induction (Supplemental Figure 12, F and G), indicating that autophagy deficiency in hepatocytes was sufficient to drive the tumorigenesis. Thus, the kinetics link the early release of HMGB1 to the later development of DR and tumorigenesis.

Active release of hepatic HMGB1 requires NRF2 in autophagydeficient livers. The above kinetics data also suggested that HMGB1 in autophagy-deficient mice might not be due to cellular death. In addition, there was very low caspase activity and no release of heat shock proteins, which are DAMP molecules commonly released from dead cells (Supplemental Figure 13, A and B). Furthermore, we detected proliferation signals (PCNA) in hepatocytes that had released HMGB1 (Supplemental Figure 13C), indicating their viability. Since HMGB1 can be actively released from viable monocytes and macrophages (11-13), we first examined whether NRF2 could be involved in the active release of HMGB1 from hepatocytes, given its important roles in the autophagy deficiency-related liver phenotype $(17,18)$. Indeed, we found that codeletion of $N r f 2$ in $A t g 7^{\text {AHep }}$ or $A t g 5^{\text {AHep }}$ mice prevented the nuclear loss of HMGB1 in autophagy-deficient hepatocytes and returned serum HMGB1 to normal levels (Figure 7, A and B, and Supplemental Figure 14, A-C).

Correspondingly, codeletion of $\mathrm{Nrf2}$ in $\mathrm{Atg} 7^{\text {मHep }}$ or Atg5 ${ }^{4 \mathrm{Hep}}$ mice completely suppressed DR and the expansion of DCs/HPCs (Figure 7, A and B, and Supplemental Figure 14, D and E). Furthermore, codeletion of Nrf2 in Atg7t ${ }^{\text {AHep }}$ mice inhibited tumor development completely (Figure 5A), as reported previously $(17,19,20)$. Since both DR and tumorigenesis were also affected by HMGB1, NRF2 probably regulated these events through the control of HMGB1 release.

The DDC diet also caused NRF2 activation (Supplemental Figure 15A) and slightly elevated LC3-II levels, the latter of which could be due to autophagy inhibition, as cotreatment of chloroquine did not further elevate LC3-II levels (Supplemental Figure 15, A and B). Consistently, we detected increased levels of high-molecular-weight aggregated p62 (Supplemental Figure 15, A-C), which might have been responsible for the elevated NRF2 activation (18) in addition to other potential mechanisms. Notably, NRF2 deletion did not completely inhibit HMGB1 release in DDC-treated mouse livers (Supplemental Figure 15, D-F). The results thus suggested that the DDC diet could cause NRF2-independent release of HMGB1. Consistently, we found that DDC-induced DR was not inhibited in Nrf2-deficient livers (Supplemental Figure 15, G and H).

NRF2 activation alone is sufficient to cause HMGB1 release but insufficient for $D R$. We next asked whether NRF2 activation alone was sufficient to cause HMGB1 release and DR, without causing liver injury and/or autophagy deficiency. Bardoxolone methyl (BM), also known as CDDO-Me or RTA 402, is a potent synthetic triterpenoid derivative that binds to KEAP1 and disrupts its interaction with NRF2, causingNRF2 nuclear translocation and activation (41).

We performed studies using several BM treatment regimes (Supplemental Figure 16A). In general, treatment with BM for up to 50 days had a minor effect on liver weights (Supplemental Figure 16B) but did not cause liver injury (Figure 7C). Systemic administration of BM for 4 or 6 days was sufficient to induce NRF2 activation in the liver and in other tissues (Figure 7D and Supplemental Figure 16, C-E). HMGB1, but not p62, was significantly reduced in the livers of BM-treated WT mice (Figure 7, D and E, and Supplemental Figure 16C), but not in the livers of BMtreated $\mathrm{Nrf2}^{-/-}$mice (Figure 7E). Concomitantly, we found that serum levels of HMGB1 were elevated in BM-treated WT mice, but not in BM-treated Nrf2-- mice (Figure 7F). We also observed this close relationship of HMGB1 release and NRF2 activity with the different treatment regimes (Supplemental Figure 16C). NRF2 activation subsided and HMBG1 levels rebounded after a 6-day BM treatment that was followed by a 16-day rest without BM treatment (22-day regime). But if BM treatment was continued 
after the first 6 daily treatments (6-day plus 14-day regime), we observed sustained NRF2 activation along with HMGB1 release. Hepatocytes were much more sensitive to BM treatment in terms of HMGB1 release, whereas other tissues did not have significant HMGB1 release with the 6-day regime (Supplemental Figure 16, E and F). These findings indicated that systemic NRF2 activation by a pharmacological agent could induce NRF2-dependent HMGB1 release without causing tissue injury or autophagy deficiency, indicating active secretion of HMGB1 from the affected hepatocytes.

Surprisingly, BM-treated livers did not develop DR or expansion of $\mathrm{CK}_{19}{ }^{+}$DCs despite the release of HMGB1, even in the long-term 50-day treatment (Figure 7G and Supplemental Figure 16G). This was surprising, because, given our study of Atg $7^{\text {Hep-ERT2 }}$ mice (Figure 6, F-H), a period of 30 days after inducing Atg7 deletion (i.e., NRF2 activation) would be sufficient for DR to develop. Taking into consideration that BM stimulation might not be equivalent to the genetic activation of NRF2, as in the autophagydeficient livers, we used a mouse strain (caNrf2) that expresses constitutively activated NRF2 in the liver under control of the Alb-Cre promoter (42). Constitutive activation of NRF2 was made possible through deletion of the Neh2 domain of NRF2, disabling its interaction with KEAP1 (42). These mice developed normally, without liver injury (42). We found that the caNrf2 liver histology was normal, with no apparent DR or DC/HPC expansion, and yet no nuclear HMGB1 (Figure 7, H and I). Taken together, these results suggest that NRF2 activation and HMGB1 release alone were not sufficient for DR to occur.

We next determined whether NRF2 activation plus a common hepatic insult could cause DR. We found that the combination of a 30-day BM treatment following administration of a commonly used hepatic carcinogen, diethylnitrosamine (DEN), given at the adult (Supplemental Figure 17, A-D) or neonatal (Supplemental Figure 17, E-G) stage, did not yield DR. Likewise, treatment of caNrf2 mice with $\mathrm{CCl}_{4}$ every 3 days for 45 days also did not lead to DR, despite a notable fibrotic response (Supplemental Figure 18A). In both cases, HMGB1 was released (Supplemental Figure 17, B, C, and F, and Supplemental Figure 18B). Interestingly, $\mathrm{CCl}_{4}$ alone was able to trigger a noticeable level of HMGB1 release (Supplemental Figure 18B), which may be due to the ability of $\mathrm{CCl}_{4}$ to activate NRF2 (43). In all cases, the liver injury was modest, with an alanine aminotransferase (ALT) level below 70 IU/1 (Supplemental Figure 17, D and G, and ref. 42). Thus, minor injury, together with NRF2 activation, was not able to drive DR, despite the release of HMGB1.

Active release of HMGB1 in autophagy-deficient livers requires the participation of inflammasomes. Monocytes and macrophages can actively secrete HMGB1 via inflammasome-mediated mechanisms $(12,13)$. The common effector molecule of the inflammasome is caspase-1, which is required for the release of HMGB1, IL-1 $\beta$, and IL-18 (11-13). We found that codeletion of Casp1 in the Atg $7^{\text {4Hep }}$ liver suppressed nuclear HMGB1 loss without affecting p62 accumulation or NRF2 activation (Figure 8A). Hepatocyte nuclear HMGB1 was retained, and serum HMGB1 remained at the basal level in $A t g 7^{\text {मHер }} \mathrm{Casp1}^{-/-}$mice (Figure 8, B-D). Notably, codeletion of Casp1 did not affect hepatomegaly or liver injury (Figure 8, E and F), indicating the presence of injury-independent mechanisms of HMGB1 release. Consistent with the impor- tance of caspase- 1 in this model, the NRF2 activator BM could not induce HMGB1 release in Casp1 $1^{-/-}$mice either (Figure 7, $\mathrm{E}$ and F).

Functionally, codeletion of Casp1 caused a dramatic reduction in DR (Figure $8 \mathrm{G}$ ) and in the expansion of CK- $19^{+}$or SOX9 ${ }^{+}$DCs/HPCs in autophagy-deficient livers (Figure $8 \mathrm{H}$ and Supplemental Figure 19A), without a significant impact on inflammation or fibrosis (Supplemental Figure 19, B and C). Our findings in $A t g 7^{4 \text { Hеp }} \mathrm{Casp1}^{-1-}$ mice were consistent with those from our studies of $A t g 7^{4 \text { Hep }} \mathrm{Nrf}^{--}$and Atg $7^{\text {AHep }} \mathrm{Hmgbl}^{-/-}$mice and suggest that the inflammasome was activated by NRF2, which in turn caused HMGB1 release and DR but not other pathologies. Indeed, we found that expression of Casp1 (Figure 8I), but not of Il1b or Il18 (Supplemental Figure 20, A and B), was elevated in autophagy-deficient livers in a NRF2-dependent manner, supporting the above hypothesis and implying a more specific role of caspase-1 in HMGB1 release but not in IL-1 $1 \beta$ nor IL-18 release in the autophagy-deficient setting.

Interestingly, we found that expression levels of Casp11 (Figure 8J and Supplemental Figure 20C), but not of Casp2, Casp3, or Casp9 (Supplemental Figure 20, D-F), were also elevated in Atg $7^{4 \mathrm{Hep}}$ livers in an NRF2-dependent manner. Both caspase-1 and caspase-11 were activated, as indicated by the appearance of the activated subunits in $\mathrm{Atg} 7^{\Delta \mathrm{Hep}}$ livers, but not in $\mathrm{Atg} 7^{\Delta \mathrm{Hep}} \mathrm{Nrf2^{-/- }}$ livers (Figure $8 \mathrm{~K}$ and Supplemental Figure 21A). Cytosolic YVADase activity (mainly caspase-1 activity) (Figure 8L and Supplemental Figure 21B) and LLSDase activity (mainly caspase-11 and caspase-1 activity) (Figure 8M) levels were elevated in Atg $7^{4 \mathrm{Hep}}$ livers and were abolished by codeletion of Nrf2 or Casp1, but not by codeletion of Hmgb1, in the autophagy-deficient livers. The LLSD sequence was derived from murine gasdermin $\mathrm{D}$, a recently identified substrate of caspase- 1 and caspase-11 $(44,45)$.

The coexistence of caspase- 1 and caspase- 11 activation in autophagy-deficient livers in our study was notable, since previous studies showed that caspase- 1 could be activated noncanonically in macrophages by caspase- 11 , which triggered IL- $1 \beta$ release via caspase-1/-11 cleavage of gasdermin $\mathrm{D}$, forming transmembrane pores composed of cleaved N-terminal gasdermin $\mathrm{D}$ fragments (46-48). Consistent with the elevated LLSDase activity, cleaved gasdermin D fragment levels were increased in $A t g 7^{4 \text { Hep }}$ livers in a NRF2-dependent manner (Figure 8K). On the other hand, expression of gasdermin $\mathrm{D}$ and other related gasdermin family proteins was not elevated in these livers (Supplemental Figure 20G). These data support a model in which NRF2 activates the caspase-11 and caspase- 1 inflammasome to promote gasdermin D cleavage and gasdermin D pore formation, leading to HMGB1 release from autophagy-deficient hepatocytes (Figure $8 \mathrm{~N}$ ).

In contrast to the autophagy deficiency, deletion of Casp1 only resulted in an incomplete reduction of HMGB1 release (Supplemental Figure 22, A-C) and did not inhibit DR (Supplemental Figure 22, D and E) in the DDC diet model. Elevation of YVADase or LLSDase activity was not evident (Supplemental Figure 22, F and $\mathrm{G})$. The presence of an inflammasome-independent mechanism for DDC diet-triggered HMGB1 release and subsequent DR was consistent with the presence of the NRF2-independent mechanisms described above (Supplemental Figure 15). It is possible that this mechanism was related to cell breakdown, as both cytosolic HSP9O and HSP70 levels were reduced in DDC-treated livers (Supplemental Figure 22H) and were not affected in autophagy- 
deficient livers (Supplemental Figure 13B). We detected no reduction in TUNEL levels in Casp1 $1^{-/}$or $\mathrm{Nrf2}^{-/-}$livers following DDC diet treatment (Supplemental Figure 22I) but found that serum ALT levels were elevated (Supplemental Figure 22J). These results suggest that, while HMGB1 was still important for DDC diet-induced DR, HMGB1 release could also be due to NRF2/inflammasomeindependent mechanisms, such as cellular breakdown.

\section{Discussion}

Loss of autophagy function triggers active HMGB1 release from hepatocytes. HMGB1 is usually released from disintegrated dead cells in a passive process (8). However, monocytes and macrophages are able to actively secrete HMGB1 (11-13). We report here that hepatocytes can release HMGB1 actively and independently of cell death. Several lines of evidence support this: (a) Acetylated forms of HMGB1 can be found in the blood of autophagydeficient mice; (b) Atg $7^{\text {Hep-ERT2 }}$ mice release HMGB1 almost immediately after Atg7 deletion, before the development of liver injury; (c) hepatocytes releasing HMGB1 can also express proliferation markers such as PCNA; (d) HMGB1 release from hepatocytes depends on NRF2 and can be induced by BM, an NRF2 activator, or induced in caNrf2 mice in which NRF2 is constitutively activated; both conditions do not cause liver injury; and (e) disruption of the Casp1-engaged inflammasomes blocks HMGB1 release but does not affect liver injury.

Several forms of posttranslational modifications, such as phosphorylation, methylation, and acetylation can result in accumulation of HMGB1 in the cytosol (49). HMGB1 modification occurs in immune cells (49) and is implicated in the translocation of HMGB1 in hepatocytes from ethanol-fed mice (16). Acetylation was shown to be required for HMGB1 to be shuttled out of the nucleus to the cytosol before its release into the extracellular space (50), thus representing an active process. On the other hand, HMGB1 passively released from dead cells is not acetylated (8). With the codeletion of Nrf2, nucleus-cytosol translocation of HMGB1 and release of HMGB1 were inhibited in autophagy-deficient hepatocytes. NRF2 activation in autophagy-deficient hepatocytes is well documented (18), but how NRF2 may promote HMGB1 translocation is not known. We speculate that NRF2 may affect the posttranslational modification of HMGB1-or that NRF2 activation may alter the nuclear structure to affect HMGB1 binding to DNA.

Active secretion of HMGB1 by macrophages or monocytes is proposed to be mediated by a nonclassical mechanism (11), as HMGB1 does not contain a secretion leader sequence. Inflammasomes may be critically involved $(12,13)$. In activated macrophages, dsRNA-dependent protein kinase (PKR) is important for triggering inflammasome/caspase-1-mediated secretion of HMGB1 (13). Our studies indicated that the active release of HMGB1 from hepatocytes can also involve the inflammasome component caspase-1. In macrophages, caspase- 1 can be activated by the canonical pathways involving several different inflammasome components but can also be activated by caspase- 11 via a noncanonical pathway (46-48). Upregulated expression of caspase-11 appeared to be essential for this pathway, which was stimulated by LPS. Caspase-11 and caspase- 1 were significantly upregulated in the autophagydeficient livers in a NRF2-dependent manner. Further studies are needed to determine whether NRF2, perhaps together with other factors, transcriptionally affects the expression of these caspases in autophagy-deficient livers.

Caspase- 1 and caspase- 11 can cleave gasdermin D to form transmembrane pores to release IL- $1 \beta$ from macrophages (44, 45). Active HMGB1 release has been known to correlate with the release of IL-1 $\beta(12,13)$ and can thus be dependent on gasdermin $\mathrm{D}$-formed pores. We detected increased proteolytic activity against gasdermin $\mathrm{D}$ in autophagy-deficient livers in a NRF2dependent manner. Overall, these data support a working model, in which NRF2 activation causes upregulation and activation of caspase- 1 and caspase-11, which leads to cleavage of gasdermin $\mathrm{D}$ and the formation of gasdermin D pores for HMGB1 release from hepatocytes. This hypothesis has yet to be fully examined using additional genetic models to assess the independent role of caspase-11 and gasdermin D.

It should be pointed out that NRF2-promoted HMGB1 release may work in a cell-dependent fashion. Mechanisms acting in one type of cell may not be fully accountable for HMGB1 dynamics in another cellular system. Hepatocytes can be particularly susceptible to the NRF2/inflammasome pathway for HMGB1 release.

HMGB1-mediated DR may represent a repair and regeneration response to liver injury. DR has been observed in many different liver injury conditions with diverse etiologies $(21,22)$ and has also been viewed as a process of liver repair and regeneration in response to chronic liver injury (23), which can also be pathogenically associated with fibrosis (22). DR reflects the expansion of DCs/HPCs from the portal region to the parenchyma. The origin and nature of these cells are still controversial, despite the fact that they usually carry biliary epithelial markers. DCs/HPCs have been implicated in compensatory epithelial hyperplasia and in the regeneration of hepatocytes or cholangiocytes that are important for hepatic regeneration and repair $(23,51,52)$, although this may not always be demonstrated in every model system (53).

As a key DAMP molecule, extracellular HMGB1 is often associated with injury, inflammation, fibrosis, and notably repair $(5-7,10,15,16)$. However, in liver injury caused by autophagy deficiency, hepatocyte-derived HMGB1 was dispensable for sterile inflammation or fibrosis, but was required for DR. Inflammation and fibrosis were both still present in the absence of HMGB1 in autophagy-deficient livers, which, however, may not necessarily indicate that HMGB1 was not involved in the process, but suggests that there could be multiple redundant mechanisms. Thus, the impact of HMGB1 on DC/HPC expansion may be mostly related to their roles in tissue repair $(6,51,52)$ and not in inflammation or fibrosis. Consistent with this thought, HMGB1 was also found to be important for regeneration but not inflammation in a model of spinal cord injury (54).

Understanding the signaling pathways that are involved in DC/HPC activation, proliferation, and differentiation is important for gaining insight into the biology of DCs/HPCs and their role in liver pathology and regeneration. However, little is known about the signals that drive DR or that promote DC/HPC expansion. TNF-like weak inducer of apoptosis (TWEAK) and FGF7 have been shown to induce mitogenesis in DCs/HPCs and to be involved in DR in injured livers $(55,56)$. However, the long-term effects of these factors were not clear. Hedgehog signaling has also been implicated in DR $(37,38)$. We found that HMGB1 was 
required for DC/HPC expansion in autophagy deficiency and had a long-term impact. We did not detect DR in Atg7 Hmgb1 ${ }^{1 \mathrm{Hep}}$ mice, even at 9 months of age. Furthermore, we detected an increase in Hedgehog signaling in autophagy-deficient livers. Thus, HMGB1 may be complementary to Hedgehog signaling or to TWEAK/ FGF7 in activating DR in autophagy-deficient livers.

HMGB1 acts as an extrinsic mechanism for DC/HPC expansion and requires its receptor RAGE. RAGE is expressed on a variety of cell types that have been shown to participate in HMGB1mediated effects $(57,58)$. HMGB1 interaction with RAGE can mediate cell proliferation, growth, and migration by activating intracellular signals such as NF- $\mathrm{BB}$ and the MAPK pathway $(57,58)$. We found that autophagy deficiency-induced DCs/HPCs expressed RAGE and CK19 and responded to dsHMGB1 with increased ERK signaling. Similar observations have been made in $M d r 2$-deficient livers and in mice fed a CDE diet. In both cases, DR was also dependent on RAGE (35). Thus, it may be assumed that, unlike the case in which HMGB1 interacts with TLRs (TLR2, TLR4, etc.) on inflammatory/fibrotic cells to drive inflammation and fibrosis, HMGB1 interacts with RAGE on DCs/HPCs in autophagy-deficient livers to drive the DR that may represent the repair process $(6,54)$.

It is important to note that our studies also indicate that HMGB1 may not work alone to promote DC/HPC expansion, because activation of NRF2 alone can cause HMGB1 release but not DR. NRF2 activation and/or HMGB1 may be necessary but insufficient for DR in autophagy-deficient livers (Figure $8 \mathrm{~N}$ ). There are probably other signals that facilitate the expansion of DCs/HPCs. Factors such as TWEAK and FGF7 $(55,56)$ and events such as Hedgehog signaling $(37,38)$ may be concomitantly needed. Another key factor may be liver injury stimulation, which occurs in autophagy-deficient livers and may trigger the above-mentioned mechanisms $(21,22)$. The simple combination of NRF2 activation (chemical or genetic) and common liver insults (DEN or $\mathrm{CCl}_{4}$ ) did not result in DR, possibly because such combinations may not cause the necessary signaling events. Future work should be directed at defining the other conditions under which DR occurs in the autophagy deficiency setting.

Toxic diets, such as those containing DDC or CDE, are well known for their ability to cause liver injury and DR $(24,25)$. This study shows that the HMGB1/RAGE axis is also an important mechanism for inducing DR following treatment with a DDC or CDE diet but that HMGB1 release may not be entirely controlled by the NRF2/inflammasome pathway in this setting. Although a DDC diet caused some autophagy deficiency and NRF2 activation, the latter was not potent enough, or needed additional cofactors not available with DDC treatment, to activate caspase- 1 and caspase-11. In addition, we found that deletion the Nrf2 or Casp1 gene was unable to completely block HMGB1 release and DR, suggesting the presence of cellular breakdown. We observed a concomitant loss of other intracellular DMAP molecules in WT mice fed a DDC diet. Together, these data suggest a dominant presence of passive HMGB1 release as a result of cellular breakdown. Nevertheless, despite the difference in the way in which HMGB1 is released, HMGB1 is as important for DR in toxic diet treatments as it is in autophagy deficiency.

Controlling HMGB1 dynamics can be an important mechanism in autophagy-mediated suppression of tumor development. Auto- phagy is an important mechanism regulating tumorigenesis $(2,59)$. Although autophagy provides a prosurvival anti-stress and nutrient-sustaining mechanism in cancer cells, it serves to suppress tumorigenesis in normal cells. There may be multiple mechanisms - such as removing damaged mitochondria and controlling oxidative stress - by which autophagy can achieve this tumor-suppressing effect. Deletion of autophagy genes in the liver leads to the development of adenoma, for which NRF2 is a critical initiator $(17,19,20)$. Accumulation of p62 in autophagy-deficient livers drives NRF2 activation, which can lead to metabolic reprogramming and $\mathrm{mTORC} 1$ activation and thereby contribute to tumor initiation $(19,39,60)$.

The present study suggests that controlling HMGB1 release can be another important mechanism in autophagy-mediated tumor suppression. Our study suggests that this effect of HMGB1 was related to the progression, but not initiation, of the tumor, since deletion of HMGB1 did not eliminate the tumors, but significantly delayed their development or metabolic reprogramming is consistent with the notion that HMGB1 is involved in processes other than tumor initiation.

The role of HMGB1 in tumor development has been studied in different contexts $(32,33)$. As a key DAMP molecule, HMGB1 is commonly associated with tissue injury, inflammation, and fibrosis $(5,6)$, and, given the strong evidence supporting a relationship between inflammation and cancer, HMGB1 may be implicated in cancer development via these mechanisms $(3,4)$. However, in autophagy-deficient livers, HMGB1 may promote tumor progression via a mechanism independent of its usual role in injury, inflammation, and fibrosis, as we did not observe a significant impact of HMGB1 deletion on these processes.

Since both hepatic tumor development and DR are promoted by HMGB1 and RAGE in autophagy-deficient livers, the question arises whether there are any connections between the 2 events. Notably, RAGE is also simultaneously involved in these 2 events in Mdr2-deficient livers (35). While DCs/HPCs have been speculated to be involved in hepatic tumor development, lineage-tracing studies using a number of hepatocellular carcinoma models were unable to show that tumor cells could be derived from these cells (31). Thus, if HMGB1-mediated DC/HPC expansion is related to HMGB1-mediated tumor progression, these 2 processes may be indirectly associated. A potential mechanism is that DCs/HPCs may affect the tumor microenvironment, e.g., matrix-cell interaction, which is related to hepatic repair and may thus also affect tumor development. Alternatively, the 2 HMGB1-regulated processes may represent a common growth factor-like effect of HMGB1 on different types of cells in the injured liver. These possibilities will need to be examined in future studies.

This study reveals the importance of HMGB1 in mediating 2 critical hepatic pathogenic processes in injured liver under autophagydeficient conditions. Our study provides mechanistic insights into DC/HPC expansion and how autophagy may suppress tumor development in the liver via the control of HMGB1 release.

\section{Methods}

Animals. Alb-Cre:Atg7 mice (18), Alb-Cre:Atg5 mice (20), Alb-Cre:Vps34 mice (61), PEPCK (phosphoenolpyruvate carboxykinase)-Cre Atg7 mice (62), Alb-Cre:hmgb1 mice (63), Rage-deficient mice (64), caNrf2 mice 
(42), and the corresponding floxed control mice have been described previously. Alb-Cre:Atg5 mice (Atg5 flox, B6.129S-Atg5 $5^{\text {tmIMyok }}$ ) were purchased from the RIKEN BioResource Research Center. Casp1-deficient mice (B6N.129S2-Casp1 $1^{\mathrm{tm} 1 \mathrm{Flv}} / \mathrm{J}$, stock no. 016621) and Nrf2-deficient mice (B6.129X1-Nfe2l2 ${ }^{\text {tmlYwk }} / \mathrm{J}$, stock no. 017009) were purchased from The Jackson Laboratory. The liver-specific inducible Atg7-deficient mice (Atg $\left.7^{\text {Hep-ERT2 }}\right)$ were generated by crossing $A t g 7^{f / f l}$ mice with $A l b$ $C r e-E R^{T 2}$ mice (40). Deletion of $A t g 7$ was induced by s.c. administration of tamoxifen $(6 \mathrm{mg} /$ day, $\times 2$ days). Strains with multiple gene alterations were created by crossbreeding. Mice were maintained on a 12-hour light/12-hour dark cycle with free access to food and water. A mixture of male and female mice were used at the ages indicated in the figure legends.

Antibodies and chemicals. The antibodies used in this study are listed in Supplemental Table 1. Tamoxifen (Sigma-Aldrich) was diluted in corn oil. Bardoxolone methyl (Cayman Chemical) was dissolved in DMSO, diluted in corn oil, and administrated i.p. (10 mg/kg). EP (Acros Organics, Thermo Fisher Scientific) was administered i.p. (40 mg/kg) twice a week for 2 or 4 weeks. The control mice received the vehicles.

Cell preparation and culture. Primary hepatocytes and nonparenchymal cells were isolated from mice using a collagenase-based standard reverse perfusion procedure. Hepatocyte purity exceeded $95 \%$ as assessed by light microscopy, and viability was typically greater than $80 \%$ as determined by a trypan blue exclusion assay. Nonparenchymal cells were fractionated using a multilayer discontinuous Nycodenz density gradient $(11 \%, 13 \%, 15 \%, 19 \%$, and $26 \%)$ as previously described (24). F1, F2, F3, and F4 cells were isolated from the interface between $11 \%$ and $13 \%, 13 \%$ and $15 \%, 15 \%$ and $19 \%$, and $19 \%$ and $26 \%$, respectively, after centrifugation at $1,500 \mathrm{~g}$ for 1 hour. These cells were then cultured overnight in William's E medium supplemented with $10 \% \mathrm{FBS}$, EGF $(10 \mathrm{ng} / \mathrm{ml})$, insulin $(10 \mu \mathrm{g} / \mathrm{ml})$, glutamine $(200 \mathrm{mM})$, and a penicillin-streptomycin combination before they were washed and subjected to experimental conditions. The progenitor cell line BMOL was obtained from G.C. Yeoh (The University of Western Australia, Crawley, Australia) (28), and cultured in the same medium as the primary cells.

Biochemical analysis. Serum levels of ALT, aspartate aminotransferase (AST), and alkaline phosphatase (ALP) were measured using commercially available kits from Pointe Scientific according to the manufacturer's protocol. Serum levels of HMGB1 were analyzed using an ELISA kit (Aviva Systems Biology) according to the manufacturer's instructions. Measurement of 4-hydroxyproline in liver tissues was performed using a hydroxyproline colorimetric assay kit (Biovision) according to the manufacturer's instruction.

Caspase assay. Caspase activity was assessed by the cleavage of site-specific reporter substrates. Total liver lysate equivalent to 20 to $40 \mu \mathrm{g}$ protein were incubated at $37^{\circ} \mathrm{C}$ with 20 to $50 \mu \mathrm{M}$ peptide substrates in caspase assay buffer (20 mM PIPES, pH 7.2, $100 \mathrm{mM} \mathrm{NaCl}$, $1 \mathrm{mM}$ EDTA, $0.1 \%$ CHAPS, $10 \%$ sucrose, $2 \mathrm{mM} \mathrm{DTT}$ ) for 1 to 16 hours. Ac-DEVD-AFC, Ac-IETD-AFC, Ac-LEHD-AFC, Ac-LLSD-AFC, and Ac-YVAD-AFC were the fluorogenic substrates used for the caspase-3, caspase 8 , caspase-9, caspase-11, and caspase-1 activity assays, respectively. Cleavage by caspases was detected at the excitation/emission wavelength of $400 \mathrm{~nm} / 505 \mathrm{~nm}$ by a fluorescence spectrometer (Tecan Infinite M200 Pro). Fluorescence readings were normalized to the background and standardized to the amount of protein analyzed.

RNA isolation and quantitative real-time PCR analysis. Total RNA was extracted from liver samples using a GeneTET RNA Purifica- tion Kit (Thermo Fisher Scientific) according to the manufacturer's protocol. cDNA was synthesized from $1 \mu \mathrm{g}$ total RNA using a M-MLV Reverse Transcriptase Enzyme System (Life Technologies, Thermo Fisher Scientific) and OligodT primers. qPCR was performed using SYBR Green Master Mixes on a 7500 FAST Real-Time PCR System (Life Technologies-Applied Biosystems, Thermo Fisher Scientific). Gene expression was calculated using the $2^{-\Delta \Delta C t}$ method and normalized to the housekeeping gene $A c t b$. Gene-specific primers and their sequences are shown in Supplemental Table 2.

Immunoblotting. Immunoblot analysis was conducted as previously described (17). The images were digitally acquired with a Kodak Image Station 4000 (Carestream Health Inc.) or a Bio-Rad ChemiDoc Image System with the corresponding software. Densitometric analysis was performed using the companion software, and the values were normalized to the loading control ( $\beta$-actin or GAPDH) and then converted to units relative to the control.

Immunofluorescence microscopy. Paraffin sections were subjected to antigen retrieval using citrate buffer ( $\mathrm{pH}$ 6.0) after deparaffinization. Slides were permeabilized and blocked with $5 \%$ goat or donkey serum in PBS containing 0.1\% Triton X (PBS-Tx) and glycine for 1 hour and then incubated overnight at $4^{\circ} \mathrm{C}$ with primary antibodies diluted in PBS. Sections were washed in PBS-Tx, following by incubation with fluorochrome-conjugated secondary antibodies. Hoechst $33342(1 \mu \mathrm{g} / \mathrm{ml})$ was used for staining of nuclei. Images were obtained using a Nikon Eclipse TE 200 epi-immunofluorescence microscope and the companion NIS-Elements AR3.2 software.

Histological analysis. Formalin-fixed mouse tissue was processed and stained with H\&E, Picrosirius red, or trichome or was stained for F4/80, CD3, CD45, or MPO for macrophages, T cells, B cells, and neutrophils, respectively, at the Indiana University Health Pathology Laboratory. Images were obtained using a Nikon Eclipse E200 microscope equipped with a SPOT RT Slider color digital camera (Diagnostic Instruments).

Statistics. Data are presented as the mean \pm SEM. The number of mice in individual experiments is shown in the figure legends. Statistical analysis was performed using SigmaStat 3.5 (Systat Software). $P$ values from at least 3 independent determinations or samples were calculated using a 2-tailed Student's $t$ test (for paired group comparisons) or 1-way ANOVA with the appropriate post hoc analysis (for multigroup comparisons). The statistical analysis methods chosen were appropriate for distribution fitting and variance. $P$ values of less than 0.05 were considered statistically significant.

Study approval. All animal experiments were approved by the IACUC of Indiana University.

\section{Author contributions}

$\mathrm{BK}$ designed research studies and wrote the manuscript. BK, $\mathrm{NH}$, $\mathrm{XC}$, and YL conducted experiments, acquired data, and analyzed data. GD, WXZ, UAK, SW, TDO, and ZD provided reagents, samples, and/or mouse strains. XMY designed research studies, analyzed data, and wrote the manuscript.

\section{Acknowledgments}

We would like to thank Eugene B. Chang (University of Chicago and the Digestive Disease Research Core Center, Chicago, Illinois, USA, sponsored by NIDDKP30 DK42086) for the Hmgb1 ${ }^{4 \text { Hep }}$ mice; Komatsu Masaaki (Niigata University Graduate School 
of Medical and Dental Sciences, Niigata, Japan) for the Atg $7^{f / f l}$ mice; Pierre Chambon and Daniel Metzger (University of Strasbourg, Strasbourg, France) for the Alb-Cre-ER ${ }^{T 2}$ mice; Wen-Xing Ding (University of Kansas Medical Center, Kansas City, USA) for the Atg5/Nrf2 mouse samples; and Valentina Factor (Laboratory of Chemical Carcinogenesis, NIH, Bethesda, Maryland, USA) for the A6 antibody. The CK19 monoclonal antibodies were obtained from the Developmental Studies Hybridoma Bank, created by the National Institute of Child Health and Human Devel- opment (NICHD), NIH, and maintained at the Department of Biology of The University of Iowa (Iowa City, Iowa, USA). This work was supported in part by NIH grants R01AA021751 and R21AA021450 (to XMY).

Address correspondence to: Xiao-Ming Yin, Department of Pathology and Laboratory Medicine, Indiana University School of Medicine, Indianapolis, Indiana 46202, USA. Phone:317.274.1779; Email: xmyin@iu.edu.
1. Ohsumi Y. Historical landmarks of autophagy research. Cell Res. 2014;24(1):9-23.

2. Mizushima N, Levine B, Cuervo AM, Klionsky DJ. Autophagy fights disease through cellular selfdigestion. Nature. 2008;451(7182):1069-1075.

3. Stauffer JK, Scarzello AJ, Jiang Q, Wiltrout RH. Chronic inflammation, immune escape, and oncogenesis in the liver: a unique neighborhood for novel intersections. Hepatology. 2012;56(4):1567-1574.

4. Kuraishy A, Karin M, Grivennikov SI. Tumor promotion via injury- and death-induced inflammation. Immunity. 2011;35(4):467-477.

5. Lotze MT, Tracey KJ. High-mobility group box 1 protein (HMGB1): nuclear weapon in the immune arsenal. Nat Rev Immunol. 2005;5(4):331-342.

6. Vénéreau E, Ceriotti C, Bianchi ME. DAMPs from cell death to new life. Front Immunol. 2015;6:422.

7. Arriazu E, et al. Signalling via the osteopontin and high mobility group box-1 axis drives the fibrogenic response to liver injury. Gut. 2017;66(6):1123-1137.

8. Scaffidi P, Misteli T, Bianchi ME. Release of chromatin protein HMGB1 by necrotic cells triggers inflammation. Nature. 2002;418(6894):191-195.

9. Wang $\mathrm{H}$, et al. HMG-1 as a late mediator of endotoxin lethality in mice. Science. 1999;285(5425):248-251.

10. Huebener P, et al. The HMGB1/RAGE axis triggers neutrophil-mediated injury amplification following necrosis. JClin Invest. 2015;125(2):539-550.

11. Gardella $\mathrm{S}$, et al. The nuclear protein HMGB1 is secreted by monocytes via a non-classical, vesicle-mediated secretory pathway. EMBO Rep. 2002;3(10):995-1001.

12. Lamkanfi M, et al. Inflammasome-dependent release of the alarmin HMGB1 in endotoxemia. J Immunol. 2010;185(7):4385-4392.

13. Lu B, et al. Novel role of PKR in inflammasome activation and HMGB1 release. Nature. 2012;488(7413):670-674.

14. Schiraldi M, et al. HMGB1 promotes recruitment of inflammatory cells to damaged tissues by forming a complex with CXCL12 and signaling via CXCR4. J Exp Med.2012;209(3):551-563.

15. Tsung A, et al. The nuclear factor HMGB1 mediates hepatic injury after murine liver ischemia-reperfusion. J Exp Med. 2005;201(7):1135-1143.

16. Ge X, et al. High mobility group box-1 (HMGB1) participates in the pathogenesis of alcoholic liver disease (ALD). J Biol Chem. 2014;289(33):22672-22691.
17. Ni HM, et al. Nrf2 promotes the development of fibrosis and tumorigenesis in mice with defective hepatic autophagy. J Hepatol. 2014;61(3):617-625.

18. Komatsu M, et al. The selective autophagy substrate $\mathrm{p} 62$ activates the stress responsive transcription factor Nrf2 through inactivation of Keap1. Nat Cell Biol. 2010;12(3):213-223.

19. Inami Y, et al. Persistent activation of Nrf2 through p62 in hepatocellular carcinoma cells. J Cell Biol. 2011;193(2):275-284.

20. Takamura A, et al. Autophagy-deficient mice develop multiple liver tumors. Genes Dev. 2011;25(8):795-800.

21. Gouw AS, Clouston AD, Theise ND. Ductular reactions in human liver: diversity at the interface. Hepatology. 2011;54(5):1853-1863.

22. Williams MJ, Clouston AD, Forbes SJ. Links between hepatic fibrosis, ductular reaction, and progenitor cell expansion. Gastroenterology. 2014;146(2):349-356

23. Miyajima A, Tanaka M, Itoh T. Stem/progenitor cells in liver development, homeostasis, regeneration, and reprogramming. Cell Stem Cell. 2014;14(5):561-574.

24. Wang X, Foster M, Al-Dhalimy M, Lagasse E, Finegold M, Grompe M. The origin and liver repopulating capacity of murine oval cells. Proc Natl Acad Sci U S A. 2003;100 Suppl 1:11881-11888.

25. Akhurst B, et al. A modified choline-deficient, ethionine-supplemented diet protocol effectively induces oval cells in mouse liver. Hepatology. 2001;34(3):519-522.

26. Ulloa L, et al. Ethyl pyruvate prevents lethality in mice with established lethal sepsis and systemic inflammation. Proc Natl Acad Sci U S A. 2002;99(19):12351-12356.

27. Shin JH, et al. Ethyl pyruvate inhibits HMGB1 phosphorylation and release by chelating calcium. Mol Med. 2015;20:649-657.

28. Tirnitz-Parker JE, Tonkin JN, Knight B, Olynyk $\mathrm{JK}$, Yeoh GC. Isolation, culture and immortalisation of hepatic oval cells from adult mice fed a choline-deficient, ethionine-supplemented diet. Int J Biochem Cell Biol. 2007;39(12):2226-2239.

29. Rountree CB, Mishra L, Willenbring H. Stem cells in liver diseases and cancer: recent advances on the path to new therapies. Hepatology. 2012;55(1):298-306.

30. Komuta M, et al. Clinicopathological study on cholangiolocellular carcinoma suggesting hepatic progenitor cell origin. Hepatology. 2008;47(5):1544-1556.

31. $\mathrm{Mu} \mathrm{X}$, et al. Hepatocellular carcinoma originates from hepatocytes and not from the progenitor/biliary compartment. J Clin Invest.
2015;125(10):3891-3903.

32. Wang $X$, et al. The role of HMGB1 signaling pathway in the development and progression of hepatocellular carcinoma: A review. Int J Mol Sci. 2015;16(9):22527-22540.

33. Yan W, et al. High-mobility group box 1 activates caspase-1 and promotes hepatocellular carcinoma invasiveness and metastases. Hepatology. 2012;55(6):1863-1875.

34. Inokuchi S, et al. Disruption of TAK1 in hepatocytes causes hepatic injury, inflammation, fibrosis, and carcinogenesis. Proc Natl Acad Sci US A. 2010;107(2):844-849.

35. Pusterla T, et al. Receptor for advanced glycation endproducts (RAGE) is a key regulator of oval cell activation and inflammationassociated liver carcinogenesis in mice. Hepatology. 2013;58(1):363-373.

36. Philips GM, et al. Hedgehog signaling antagonist promotes regression of both liver fibrosis and hepatocellular carcinoma in a murine model of primary liver cancer. PLoS One. 2011;6(9):e23943.

37. Omenetti A, Choi S, Michelotti G, Diehl AM. Hedgehog signaling in the liver.J Hepatol. 2011;54(2):366-373.

38. Jung Y, et al. Signals from dying hepatocytes trigger growth of liver progenitors. Gut. 2010;59(5):655-665.

39. Saito T, et al. p62/Sqstm1 promotes malignancy of HCV-positive hepatocellular carcinoma through Nrf2-dependent metabolic reprogramming. Nat Commun. 2016;7:12030.

40. Schuler M, Dierich A, Chambon P, Metzger D. Efficient temporally controlled targeted somatic mutagenesis in hepatocytes of the mouse. Genesis. 2004;39(3):167-172.

41. Yates MS, et al. Pharmacodynamic characterization of chemopreventive triterpenoids as exceptionally potent inducers of Nrf2-regulated genes. Mol Cancer Ther. 2007;6(1):154-162.

42. Köhler UA, et al. Activated Nrf2 impairs liver regeneration in mice by activation of genes involved in cell-cycle control and apoptosis. Hepatology. 2014;60(2):670-678.

43. Aleksunes LM, Slitt AM, Cherrington NJ, Thibodeau MS, Klaassen CD, Manautou JE. Differential expression of mouse hepatic transporter genes in response to acetaminophen and carbon tetrachloride. Toxicol Sci. 2005;83(1):44-52.

44. Kayagaki N, et al. Caspase-11 cleaves gasdermin $\mathrm{D}$ for non-canonical inflammasome signalling. Nature. 2015;526(7575):666-671.

45. Shi J, et al. Cleavage of GSDMD by inflammatory caspases determines pyroptotic cell death. 
Nature. 2015;526(7575):660-665.

46. Hagar JA, Powell DA, Aachoui Y, Ernst RK, Miao EA. Cytoplasmic LPS activates caspase-11: implications in TLR4-independent endotoxic shock. Science. 2013;341(6151):1250-1253.

47. Wang S, Miura M, Jung YK, Zhu H, Li E, Yuan J. Murine caspase-11, an ICE-interacting protease, is essential for the activation of ICE. Cell. 1998;92(4):501-509.

48. Kayagaki N, et al. Non-canonical inflammasome activation targets caspase-11. Nature. 2011;479(7371):117-121.

49. Lu B, et al. Molecular mechanism and therapeutic modulation of high mobility group box 1 release and action: an updated review. Expert Rev Clin Immunol. 2014;10(6):713-727.

50. Bonaldi T, et al. Monocytic cells hyperacetylate chromatin protein HMGB1 to redirect it towards secretion. EMBO J. 2003;22(20):5551-5560.

51. Lu WY, et al. Hepatic progenitor cells of biliary origin with liver repopulation capacity. Nat Cell Biol. 2015;17(8):971-983.

52. Raven A, et al. Cholangiocytes act as facultative liver stem cells during impaired hepatocyte regeneration. Nature. 2017;547(7663):350-354.

53. Schaub JR, Malato Y, Gormond C, Willenbring H. Evidence against a stem cell origin of new hepatocytes in a common mouse model of chronic liver injury. Cell Rep. 2014;8(4):933-939.

54. Dong Y, et al. HMGB1 protein does not mediate the inflammatory response in spontaneous spinal cord regeneration: a hint for CNS regeneration. J Biol Chem. 2013;288(25):18204-18218.

55. Jakubowski A, et al. TWEAK induces liver progenitor cell proliferation. JClin Invest. 2005;115(9):2330-2340.

56. Takase HM, et al. FGF7 is a functional niche signal required for stimulation of adult liver progenitor cells that support liver regeneration. Genes Dev. 2013;27(2):169-181.

57. Taguchi A, et al. Blockade of RAGE-amphoterin signalling suppresses tumour growth and metastases. Nature. 2000;405(6784):354-360.

58. Palumbo R, et al. Extracellular HMGB1, a signal of tissue damage, induces mesoangioblast migration and proliferation. JCell Biol.
2004;164(3):441-449.

59. Amaravadi R, Kimmelman AC, White E. Recent insights into the function of autophagy in cancer. Genes Dev. 2016;30(17):1913-1930.

60. Umemura A, et al. p62, Upregulated during preneoplasia, induces hepatocellular carcinogenesis by maintaining survival of stressed HCC-initiating cells. Cancer Cell. 2016;29(6):935-948.

61. Jaber N, et al. Class III PI3K Vps34 plays an essential role in autophagy and in heart and liver function. Proc Natl Acad Sci U S A. 2012;109(6):2003-2008.

62. Jiang M, Wei Q, Dong G, Komatsu M, Su Y, Dong Z. Autophagy in proximal tubules protects against acute kidney injury. Kidney Int . 2012;82(12):1271-1283.

63. Zhu X, et al. Cytosolic HMGB1 controls the cellular autophagy/apoptosis checkpoint during inflammation. J Clin Invest. 2015;125(3):1098-1110.

64. Constien R, et al. Characterization of a novel EGFP reporter mouse to monitor Cre recombination as demonstrated by a Tie2 Cre mouse line. Genesis. 2001;30(1):36-44. 